\title{
A general approach for the location of transfer points on a network with a trip covering criterion and mixed distances
}

\author{
M. C. López-de-los-Mozos a, Juan A. Mesa ${ }^{b}$, Anita Schöbel ${ }^{c}$ \\ ${ }^{a}$ Departamento de Matemática Aplicada I, E.T.S. de Ingeniería Informática, Avda. Reina Mercedes, s/n, 41012, Universidad de Sevilla, Spain \\ ${ }^{\mathrm{b}}$ Departamento de Matemática Aplicada II, E.T.S. de Ingeniería, Camino de los Descubrimientos, s/n, 41092, Universidad de Sevilla, Spain \\ ${ }^{\mathrm{c}}$ Institut für Numerische and Angewandte Mathematik. Universität Göttingen, Germany
}

Keywords:

Location

Networks

Covering problems

Mixed distances

\begin{abstract}
A B S T R A C T
In this paper we consider a trip covering location model in a mixed planar-network space. An embedded network in the plane represents an alternative transportation system in which traveling is faster than traveling within the plane. We assume that the demand to be covered is given by a set of origindestination pairs in the plane, with some traffic between them. An origin-destination pair is covered by two facility points on the network (or transfer points), if the travel time from the origin to destination by using the network through such points is not higher than a given acceptance level related to the travel time without using the network. The facility location problems studied in this work consist of locating one or two transfer points on the network such that, under several objective functions, the traffic through the network is maximized. Due to the continuous nature of these problems, a general approach is proposed for discretizing them. Since the non-convexity of the distance function on cyclic networks also implies the absence of convexity of the mixed distance function, such an approach is based on a decomposition process which leads to a collection of subproblems whose solution set can be found by adapting the general strategy to each problem considered.
\end{abstract}

\section{Introduction}

Given a real or virtual underlying network, the general network design problem consists of two intertwined problems (Contreras \& Fernández, 2012 ): to select from the network a number of points for siting facilities, and to interconnect these points by choosing

links of the underlying network. In our problem we have a network embedded in the plane and a set of demand points in the plane. The demand or traffic between demand points is given by an OD-matrix (Origin-Destination matrix) which is assumed to be static, and it is satisfied by traveling from one to the other point of each pair. We want to select points on the network to access/ exit to/from it. These facility points are used to transfer from the plane to the embedded given network, thus allowing the connection of the pairs of demand points by using the network. A subproblem of the general network design problem arises in the design of a railway network ( Laporte \& Mesa, 2015; Laporte, Mesa, \& Ortega, 2000 ) that consists in locating a number of stations and the tracks to connect the station-facilities.
The problem of locating stations in a railway network was indirectly tackled in Vuchic and Newell (1968), in which the optimal interstation spacing problem in a commuter line was researched. The objective of this problem is to minimize the total time of passengers going to a city center along a railway line. A commuter line competing with a freeway was considered in Vuchic (1969) in order to maximize the number of passengers on the basis of shortest travel times. The aim was also to determine the optimal interstation space between pairs of adjacent stations. Apart from the papers (Laporte, Mesa, \& Ortega, 2002; Laporte, Mesa, Ortega, \& Sevillano, 2005; Repolho, Antunes, \& Church, 2013) in which the feasible solution space for locating stations was discrete and the objectives were to maximize the passenger coverage, trip coverage and savings in travel cost, respectively, several objective functions have been considered in those models in which stations can be located along the edges of the railway network: saving in passenger travel time (Hamacher, Liebers, Schöbel, Wagner, \& Wagner, 2001), coverage/number of new stations (Schöbel, 2005), additional travel time (Schöbel, Hamacher, Liebers, \& Wagner, 2009), and total travel time (Carrizosa, Harbering, \& Schöbel, 2016). The maximal covering location problem was introduced in Church and ReVelle (1974) in which a number of facilities are to be located on a network so that the population within a service distance is 
maximized. In Murawski and Church (2009) time to access the facilities is improved by upgrading edges of the transportation network. The objective function consists in maximizing the number of people who are covered by upgrading some parts of the network. In order to locate interchanges points in a highway network a cost-benefit objective function is considered in Repolho, Church, and Antunes (2010) that takes into account a route choice model.

In the problem dealt with in this paper, there are existing demand points in the Euclidean plane whereas new facilities are to be located in a network. Instead of covering facilities the objective aims at covering trips between each pair of existing facilities. These trips can be done either by using the plane with the Euclidean distance or by a plane-network combination in which the section on the network is supposed to be traversed faster than those on the plane. Therefore, there is competition between the mode that only uses the planar distance and the combined one. If the time spent by the trip in the combined mode is lower than that of the planar mode then the OD-pair is said to be covered by the transfer points used to access/exit the network. Each pair of existing facilities has an associated demand and the objective is to maximize the number of trips for which the combined mode is preferable to the planar one.

In this paper we assume the decision space is the set of points on the edges of the network. The nature of the decision space when locating new transfer points in long and medium distance railway networks as well as in underground urban/suburban rapid transit systems is continuous. As was pointed out in Laporte, Mesa, and Perea (2014) 30\% of the stations of the Spanish high-speed network are situated outside cities in the countryside, thus allowing a continuous decision space. Furthermore, the continuous setting of the decision space allows the use of continuous optimization methods thus providing insight into the behavior of the objective function and the problem itself. Moreover, new development areas, congestion or environmental and energy consumption are reasons for constructing new stations. An example is the use of a section of the railway line Seville-Huelva as a new commuter line (Line 5) for the metropolitan area of Seville (Laporte et al., 2014). For this line new stations were built, some of them between towns. Another example is the construction of a new station in the high-speed line Madrid-Seville between Córdoba and Puertollano stations. The new station: Villanueva de Córdoba-Los Pedroches covers an area of the north of the province of Córdoba.

Since the traveling demand is given by pairs of existing points it is coherent to consider the location of pairs of transfer points acting as access/exit points on the network. Under the assumption of a network with a set of existing node-stations, there are two problems particularly relevant from an applied point of view, which deal with locating one, or two, transfer points in order to maximize the amount of OD-pairs additionally covered, that is, those OD-pairs which cannot be covered by the existing stations. In the first problem only one transfer point is located since this point in combination with each station works as an access/exit point. In the second problem, which seeks to locate two transfer points, the additional coverage refers the OD-pairs covered either by the two transfer points or by a combination of each point with some station, but not yet covered by pairs of vertices. Both problems also exclude the OD-pairs previously covered by the existing stations. Moreover, the second problem presents a more general formulation of the model, in the way that it incorporates the additional coverage provided both by the two points and by each point separately.

For solving these additional covering problems, this paper proposes a methodology which is based on considering a key problem, whose solution procedure provides a general approach which can be adapted to the remaining problems. This key problem does not require any hypothesis on the network (it is possible that there is no already located station), since it seeks to locate two transfer points maximizing the amount of OD-pairs covered. The relevance of this problem derives from it allowing the development of a theoretical framework which is shared by the above one or two transfer additional covering problems, as well as by other related problems.

Summarizing, the main contribution of this paper is, in the first place, to propose a comprehensive approach for solving several problems dealing with locating transfer points on cyclic transportation networks under several objective functions, all of them focused on covering OD-pairs instead of single demand points. Moreover, the proposed approach to solve the problem of maximizing the coverage obtained by 2-transfer points is used for the more real problems of locating one or two transfer points so that the additional coverage (in presence of already functioning stations) will be maximized. The problems studied deal with a continuous model, whose analysis enlarges the knowledge on both the geometric structure of the problems and the behavior and properties of the objective functions. On the other hand, such an approach describes a flexible methodology which, by incorporating slight modifications, allows to discretize the solution set of all problems considered.

In a previous paper (Körner, Mesa, Perea, Schöbel, \& Scholz, 2014) the key problem was solved when the new facilities are to be located in segments and tree networks. In this paper we extend the approach applied in Körner et al. (2014) to the case of general networks by taking into account the loss of convexity of the distance function between pairs of points through the network. The main aim of this research is to solve that complex continuous location problem by reducing the candidate set to a finite one from which an optimal solution is selected by means of a polynomial time algorithm.

The paper is organized as follows: after this introduction the elements of the model are presented in Section 2. The necessary definitions and results on the distance on networks are summarized in Section 3. Section 4 provides a decomposition of the first problem into two types of subproblems, such that for each subproblem a solution method is proposed in Sections 5. Sections 6 and 7 study respectively two new problems, both related to the previous one, in which one and two transfer points are located under different objective functions, and Section 8 is devoted to presenting the corresponding algorithms and discussing its computational complexity. The paper ends with some conclusions and further research.

\section{Elements of the model}

In order to formulate the problems with a mixed mode of transportation, we consider a connected network $\mathcal{N}(V, E)$ representing a high-speed system, with $|V|$ nodes and $|E|$ edges (where $|\cdot|$ denotes cardinality). We assume that the network is embedded in the Euclidean plane and that each undirected edge $e \in E$ can be modeled as a straight-line segment. The embedding of $\mathcal{N}$ in the Euclidean plane as well as the coordinates of the nodes in $\mathcal{N}$ will allow us to compute the distances along the network. More precisely, the length $l(u, v)$ of an edge (or a subedge) $[u, v]$ is the Euclidean distance $\|u-v\|$ between its endpoints, and by using shortest path algorithms we can moreover compute the distance between each pair of nodes. Moreover, the triangle inequality holds in this model.

Let $\mathcal{N}$ be the continuum set of points of the edges. The edge lengths induce a distance function $d$ such that, for any two points $x, y \in \mathcal{N}, d(x, y)$ is the length of any shortest path in $\mathcal{N}(V, E)$ connecting $x$ and $y$. If $x$ and $y$ are on the same edge, then $d(x, y)$ coincides with the length of the subedge $[x, y]$.

For $x, y \in \mathcal{N}$, the travel distance between both points is given by $\alpha d(x, y)$, with $\alpha \in(0,1)$. Parameter $\alpha$ is a speed factor, such that 

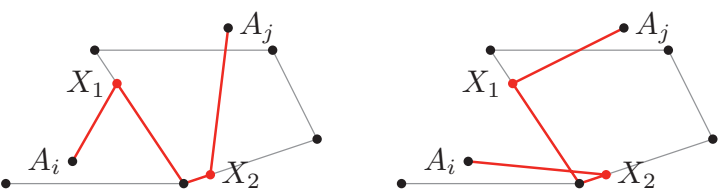

Fig. 1. Travel paths for: $h_{i j}^{+}\left(X_{1}, X_{2}\right)$ (left), and $h_{i j}^{-}\left(X_{1}, X_{2}\right)$ (right).

$\alpha d(x, y)$ represents the traveling time between $x, y$ by using the high-speed network. It can immediately be seen that, with these distance, $\mathcal{N}$ is a metric space.

- Let $\mathcal{A}=\left\{A_{i}=\left(a_{i}, b_{i}\right), i=1, \ldots, n\right\} \subset \mathbb{R}^{2}$ be a set of existing demand points on the plane. We assume that distances between two points in the plane can be estimated by the Euclidean metric.

- Let $T=\left(t_{i j}\right) \in \mathbb{R}^{n \times n}$ be an origin-destination matrix in which trip patterns are codified, i.e., $t_{i j}$ is the weight of the ordered pair $(i, j)$. This matrix is known a priori: for example, in a transportation context each $t_{i j}$ can be viewed as the number of trips from an origin $A_{i}$ to a destination $A_{j}$, and in a telecommunication setting it could represent the amount of data transferred from server $A_{i}$ to server $A_{j}$.

- Given an OD-pair $(i, j)$, the travel distance by using the network through the points $X_{1}, X_{2} \in \mathcal{N}$ (or the 2-facility point $\left(X_{1}, X_{2}\right)$ ) is obtained from the two possible travel paths: $\left(A_{i}, X_{1}, X_{2}, A_{j}\right)$, and $\left(A_{i}, X_{2}, X_{1}, A_{j}\right)$, linking origin and destination (see Fig. 1). For each of them, the travel distance is given by

$$
\begin{aligned}
& h_{i j}^{+}\left(X_{1}, X_{2}\right)=\left\|A_{i}-X_{1}\right\|+\alpha d\left(X_{1}, X_{2}\right)+\left\|X_{2}-A_{j}\right\| \\
& h_{i j}^{-}\left(X_{1}, X_{2}\right)=\left\|A_{i}-X_{2}\right\|+\alpha d\left(X_{2}, X_{1}\right)+\left\|X_{1}-A_{j}\right\| .
\end{aligned}
$$

The mixed travel distance between $A_{i}$ and $A_{j}$ by using the highspeed network through the transfer points $X_{1}, X_{2} \in \mathcal{N}$ is given by the shortest travel distance obtained from both travel paths, and it is written as:

$$
f_{i j}\left(X_{1}, X_{2}\right)=\min \left\{h_{i j}^{+}\left(X_{1}, X_{2}\right), \quad h_{i j}^{-}\left(X_{1}, X_{2}\right)\right\} .
$$

Symmetry of such paths implies $h_{i j}^{+}\left(X_{1}, X_{2}\right)=h_{j i}^{-}\left(X_{1}, X_{2}\right)$, $h_{i j}^{-}\left(X_{1}, X_{2}\right)=h_{j i}^{+}\left(X_{1}, X_{2}\right)$, and therefore $f_{i j}\left(X_{1}, X_{2}\right)=f_{j i}\left(X_{1}, X_{2}\right)$.

- Let $\widehat{D}=\left(\widehat{d}_{i j}\right) \in \mathbb{R}^{n \times n}$ be a symmetric matrix, with $0 \leq \widehat{d}_{i j}<$ $\left\|A_{i}-A_{j}\right\|$, for $i \neq j$, and $\widehat{d_{i i}}=0, i=1, \ldots, n$. The values of $\widehat{D}$ represent the acceptance levels for using the network, meaning that the OD-pair $(i, j)$ chooses the high-speed network if and only if the mixed travel distance by using it is less than or equal to $\widehat{d}_{i j}$. In other words, the OD-pairs always choose the faster option. In the following, we assume that $i \neq j$ to avoid the trivial case.

Definition 1. The OD-pair $(i, j)$ is covered by $X_{1}, X_{2} \in \mathcal{N}$ if $f_{i j}\left(X_{1}, X_{2}\right) \leq \widehat{d}_{i j}$. by

Let $C\left(X_{1}, X_{2}\right)$ be the set of O/D pairs covered by $X_{1}, X_{2}$, given

$C\left(X_{1}, X_{2}\right)=\left\{(i, j), \quad i \neq j, \quad 1 \leq i, j \leq n: f_{i j}\left(X_{1}, X_{2}\right) \leq \widehat{d_{i j}}\right\}$.

From symmetry both of the acceptance level matrix $\widehat{D}$ and the mixed travel distance we have

$(i, j) \in C\left(X_{1}, X_{2}\right)$ if and only if $(j, i) \in C\left(X_{1}, X_{2}\right)$.

As it has been pointed out in the previous section, in the first place we formulate the key problem, which presents structural properties shared by the remaining problems, and whose solution procedure provides a general methodology for analyzing and solving the one and two additional covering problems, which are formulated subsequently.

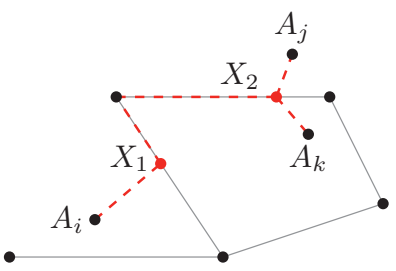

Fig. 2. Given the nonnegative acceptance levels $\widehat{d_{i j}}<\left\|A_{i}-A_{j}\right\|, \widehat{d_{i k}}<\left\|A_{i}-A_{k}\right\|$, $\widehat{d}_{j k}<\left\|A_{j}-A_{k}\right\|$, and a suitable speed factor $\alpha \in(0,1)$, we have $(i, j),(i, k) \in$ $C\left(X_{1}, X_{2}\right)$. However $(j, k) \notin C\left(X_{1}, X_{2}\right)$. The objective value at $\left(X_{1}, X_{2}\right)$ is: $F\left(X_{1}, X_{2}\right)=$ $t_{i j}+t_{j i}+t_{i k}+t_{k i}$. There may be several points $\left(X_{1}, X_{2}\right)$ with the same objective value.

1. The first objective function measures the total weight of ODpairs captured by each pair of transfer points $X_{1}, X_{2} \in \mathcal{N}$, and it is given by:

$F\left(X_{1}, X_{2}\right)=\sum_{(i, j) \in C\left(X_{1}, X_{2}\right)} t_{i j}$

In this function whether the node set $V$ contains already located stations or not is not relevant, since it seeks to compute the weight of pairs covered by the two transfer points when they assume the symmetric role of access/exit points (see Fig. 2).

The key problem, briefly the 2-transfer covering problem (2TC), is to find two transfer points $X_{1}, X_{2} \in \mathcal{N}$ such that the sum of weights of all OD-pairs covered by such points is maximized:

$\max _{X_{1}, X_{2} \in \mathcal{N}} F\left(X_{1}, X_{2}\right):=\sum_{(i, j) \in C\left(X_{1}, X_{2}\right)} t_{i j}$

2. Contrary to the above model, this problem and the following one deal with additional coverage, and they require the hypothesis of having a set of stations already located, these latter ones selected from the node set.

Without loss of generality we can assume that all nodes of $V$ are already located transfer points. From Definition 1 , the OD-pair $(i, j)$ is covered by any $X \in \mathcal{N}$ if there exists $v \in V$ such that $f_{i j}(X, v) \leq \widehat{d}_{i j}$. Thus, the set $C(X)$ containing the OD-pairs covered by $X$ is

$$
\begin{aligned}
C(X)= & \left\{(i, j), i \neq j, 1 \leq i, j \leq n: f_{i j}(X, v) \leq \widehat{d}_{i j}\right. \\
& \text { for some } v \in V\}
\end{aligned}
$$

Similarly, the set $C_{V}$ of OD-pairs already covered by the nodes of $V$ is given by

$$
\begin{aligned}
& C_{V}=\left\{(i, j), i \neq j, 1 \leq i, j \leq n: f_{i j}(w, v) \leq \widehat{d_{i j}}\right. \\
& \quad \text { for some } w, v \in V\}
\end{aligned}
$$

Thus, the second objective function can be stated as

$$
F_{V}(X):=\sum_{(i, j) \in C(X) \backslash C_{V}} t_{i j}
$$

And the 1-transfer additional covering problem (1-TAC) is:

$$
\max _{X \in \mathcal{N}} F_{V}(X):=\sum_{(i, j) \in C(X)} t_{i j}
$$

Finally, in this case two transfer points $\left(X_{1}, X_{2}\right)$ are located by addressing the additional coverage. As above, we assume that all nodes are stations, and also the set $C_{V}$ is excluded. The objective function integrates the above formulations since it takes into account both the amount of ODpairs covered by $\left(X_{1}, X_{2}\right)$ and the OD-pairs covered by each point in combination with the stations already located at 
nodes, as follows:

$$
F_{A}\left(X_{1}, X_{2}\right):=\sum_{(i, j) \in\left(C\left(X_{1}, X_{2}\right) \cup C\left(X_{1}\right) \cup C\left(X_{2}\right)\right) \backslash C_{V}} t_{i j}
$$

The 2-transfer additional covering problem (2-TAC), seeks the location of two transfer points maximizing the total weight of OD-pairs additionally covered:

$$
\max _{X_{1}, X_{2} \in \mathcal{N}} F_{A}\left(X_{1}, X_{2}\right):=\sum_{(i, j) \in\left(C\left(X_{1}, X_{2}\right) \cup C\left(X_{1}\right) \cup C\left(X_{2}\right)\right) \backslash C_{V}} t_{i j}
$$

Remark 2. Each pair of associated problems obtained by excluding, or not, $C_{V}$ from the corresponding formulation are equivalent, but not the same, in the following way: they share the same solution set (which is a straightforward consequence from the fact that $C_{V}$ is a constant set), although their optimal solutions could be different. That is, (2-TC) and $\max _{X_{1}, X_{2} \in \mathcal{N}} F\left(X_{1}, X_{2}\right):=\sum_{(i, j) \in C\left(X_{1}, X_{2}\right) \backslash C_{V}} t_{i j}$ are equivalent, and so on.

Although problems (1-TAC) and (2-TAC) present special relevance regarding the applications, the procedure for solving problem (2-TC) provides a general methodology which can be applied to all problems presented in this paper. In fact, the nature of such a methodology allows the resolution to be extended to some generalizations of these problems, as the $p$-transfer additional covering problem $(p>2)$. This motivates that problem $(2-\mathrm{TC})$ is studied in the first place. The strategy for solving this problem lies in decomposing it into a collection of subproblems such that, for each of them, a partition of the feasible solution set is constructed, and from such partition, a finite subset containing some optimal solution is selected.

Problem (2-TC) has been first solved for the particular case where $\mathcal{N}$ is a segment of a straight line, and subsequently the method was extended to the case where $\mathcal{N}$ is a tree network $\mathcal{T}$ (see Körner et al., 2014). Thus, henceforth we will consider that $\mathcal{N}$ contains at least one cycle. Under this assumption, the approach applied to the tree network case cannot be directly extended to this case due to the absence of convexity of the distance function on a cyclic network.

In order to solve this problem, we next summarize some concepts and results on distances in networks.

\section{Previous results on distances on networks}

For evaluating the objective function of problem (2-TC), it is first necessary to obtain an analytical expression for the distance between any two points $P, Q$ of the network $\mathcal{N}$. The aforementioned non-convexity leads to the fact that the expression for the distance $d(P, Q)$ could vary through the network. In this section we partition the network into a collection of subedges such that for each pair of subedges, the distance between their points can be computed. To this end, we first need to review some concepts dealing with the distance on networks.

Hereinafter we assume a network $\mathcal{N}$ with a distance function $d(\cdot, \cdot)$ such that the triangle inequality holds. Let $e=[u, w] \in E$ be an edge of $\mathcal{N}$ with length $l_{e}$, and let $P$ be a point on edge $e$. Let $x=$ $l(u, P)$ denote the length of subedge $[u, P]$, and let $l(w, P)=l_{e}-x$ denote the length of subedge $[P, w]$. It is well known that for any node $v \in V$, the distance $d(v, P)=\min \left\{d(v, u)+x, d(v, w)+l_{e}-x\right\}$ is concave and piecewise linear on $x \in\left[0, l_{e}\right]$, with at most two pieces with slopes 1 and -1 . From the triangle inequality we have $l_{e}=d(u, w) \leq|d(v, u)-d(v, w)|$, therefore the distance function $d(v, P)$ on $[u, w]$ is linear. For the sake of completeness we include the following concepts and definitions, although all of them

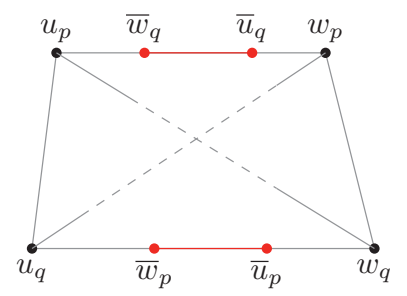

Fig. 3. Antipodal points.

can be found in Hooker, Garfinkel, and Chen. (1991) and references therein.

Definition 3. Given a point $Q \in \mathcal{N}$ and an edge $[u, w]$, the point $P \in[u, w]$ at which $d(Q, P)$ is maximized is called antipodal to $Q$ in $[u, w]$.

Definition 4. A point $\bar{v} \in[u, w]$, other than a node, is called an arc bottleneck point if there is a node $v$ for which $\bar{v}$ is antipodal to $v$. In this case, we call it the arc bottleneck point $\bar{v}$.

Clearly, the edge $e=[u, w]$ contains at most $|V|$ arc bottleneck points. If $\bar{v} \in[u, w]$ is antipodal to $v$ we have $d(v, u)+l(u, \bar{v})=$ $d(v, w)+l(w, \bar{v})$, hence such a point $\bar{v}$ can be identified by the length of the subedge $[u, \bar{v}]$, given by: $l(u, \bar{v})=\frac{d(v, w)-d(v, u)+l_{e}}{2}$.

Definition 5. Let $B_{e}$ be the set of arc bottleneck points of edge $e=[u, w]$, and let $\bar{v}, \bar{v}^{\prime}$ be two adjacent points of $B_{e} \cup\{u, w\}$. Then the closed subedge $L=\left[\bar{v}, \bar{v}^{\prime}\right]$ is called a linear arc segment (or a treelike segment (Hooker et al., 1991)).

We remark that $B_{e}$ has $O(|V|)$ elements. On each linear arc segment the distance $d(v, \cdot)$ is linear. If $B_{e}=\emptyset$ the entire edge $e=[u, w]$ (including nodes) is a linear arc segment. The set of all linear arc segments of an edge $e$ is denoted by $\mathcal{L}(e)$.

Let $\left[u_{p}, w_{p}\right]$ and $\left[u_{q}, w_{q}\right]$ be two edges of the network $\mathcal{N}$, and let $\left[\bar{w}_{p}, \bar{u}_{p}\right] \subseteq\left[u_{q}, w_{q}\right],\left[\bar{w}_{q}, \bar{u}_{q}\right] \subseteq\left[u_{p}, w_{p}\right]$ be the subedges for which the extreme points are antipodal points as follows: $\bar{u}_{p}, \bar{w}_{p}$ are the antipodal points to $u_{p}, w_{p}$, respectively, and $\bar{u}_{q}, \bar{w}_{q}$ are the antipodal points to $u_{q}, w_{q}$, respectively (see Fig. 3). From previous definitions we have $l\left(\bar{w}_{q}, \bar{u}_{q}\right)=l\left(\bar{w}_{p}, \bar{u}_{p}\right)$ and $d\left(\bar{w}_{q}, \bar{w}_{p}\right)=d\left(\bar{u}_{q}, \bar{u}_{p}\right)$ (see Hooker et al., 1991 for a more detailed explanation).

Definition 6. Let $L_{p}, L_{q}$ be two linear arc segments of different edges $\left[u_{p}, w_{p}\right]$ and $\left[u_{q}, w_{q}\right]$, respectively, and let $\left[\bar{w}_{q}, \bar{u}_{q}\right] \subseteq\left[u_{p}, w_{p}\right]$ and $\left[\bar{w}_{p}, \bar{u}_{p}\right] \subseteq\left[u_{q}, w_{q}\right]$ be the subedges for which $\bar{u}_{p}, \bar{w}_{p}$ are the antipodal points to $u_{p}, w_{p}$, respectively, and $\bar{u}_{q}, \bar{w}_{q}$ are the antipodal points to $u_{q}, w_{q}$, respectively. If $L_{p} \subseteq\left[\bar{w}_{q}, \bar{u}_{q}\right]$ and $L_{q} \subseteq\left[\bar{w}_{p}, \bar{u}_{p}\right]$, then $L_{p}, L_{q}$ are called antipodal segments to each other.

(This definition includes the case $\left[\bar{w}_{q}, \bar{u}_{q}\right]=\left[u_{p}, w_{p}\right]$ and $\left.\left[\bar{w}_{p}, \bar{u}_{p}\right]=\left[u_{q}, w_{q}\right]\right)$. The following result summarizes the behavior of distance $d(P, Q)$ on linear arc segments of different edges (see Hooker et al., 1991).

Lemma 7. Let $P$ be restricted to linear arc segment $L_{p}$ of edge $\left[u_{p}, w_{p}\right]$ and $Q$ to linear arc segment $L_{q}$ of edge $\left[u_{q}, w_{q}\right]$, with $\left[u_{p}, w_{p}\right] \neq\left[u_{q}, w_{q}\right]$.

1. If $L_{p}, L_{q}$ are antipodal segments to each other, $d(P, Q)$ is concave.

2. Otherwise, $d(P, Q)$ is linear.

In case 1 , the distance between a point $P$ on the segment $\left[\bar{w}_{q}, \bar{u}_{q}\right]$ and a point $Q$ on the segment $\left[\bar{w}_{p}, \bar{u}_{p}\right]$ behaves like the distance on the parallelogram in Fig. 3(a), and it is concave. Distance on any other pair of segments behaves like distance on a line segment and is therefore linear. 
By denoting $x=l\left(u_{p}, P\right)$, with $x \in\left[0, l\left(u_{p}, w_{p}\right)\right]$ and $y=l\left(u_{q}, Q\right)$, with $y \in\left[0, l\left(u_{q}, w_{q}\right)\right]$, we can compute the distance $d(P, Q)$ for the cases considered in this lemma.

$$
d(P, Q)=\left\{\begin{array}{c}
\min \left\{x+d\left(u_{p}, u_{q}\right)+y, \quad l\left(u_{p}, w_{p}\right)-x+d\left(w_{p}, w_{q}\right)\right. \\
\left.\quad+l\left(u_{q}, w_{q}\right)-y\right\}, \quad L_{p}, L_{q} \text { antipodal } \\
x+d\left(u_{p}, u_{q}\right)+y, \quad L_{p} \subseteq\left[u_{p}, \bar{w}_{q}\right], \quad L_{q} \subseteq\left[u_{q}, \bar{w}_{p}\right] \\
\quad \text { r } L_{q} \subseteq\left[\bar{w}_{p}, \bar{u}_{p}\right] \\
x+d\left(u_{p}, w_{q}\right)+l\left(u_{q}, w_{q}\right)-y, \quad L_{p} \subseteq\left[u_{p}, \bar{w}_{q}\right], \\
\quad L_{q} \subseteq\left[\bar{u}_{p}, w_{q}\right] .
\end{array}\right.
$$

Note that if $L_{p}, L_{q}$ are antipodal segments, with $u_{p} \neq \bar{w}_{q}, \bar{u}_{q} \neq w_{p}$ and similarly $u_{q} \neq \bar{w}_{p}, \bar{u}_{p} \neq w_{q}$, then the distance $d(P, Q)$ can also be computed by $\min \left\{x+d\left(u_{p}, w_{q}\right)+l\left(u_{q}, w_{q}\right)-y, l\left(u_{p}, w_{p}\right)-x+\right.$ $d\left(w_{p}, u_{q}\right)+y$ \}. Finally, the remaining cases obtained by combining $L_{p}, L_{q}$ can be reduced to one of these by symmetry (for example, for the case $L_{p} \subseteq\left[u_{p}, \bar{w}_{q}\right], L_{q} \subseteq\left[\bar{u}_{p}, w_{q}\right]$ the distance can also be equivalently obtained as $\left.l\left(u_{p}, w_{p}\right)-x+d\left(w_{p}, u_{q}\right)+y\right)$.

We now comment the case in which $P, Q$ lie on the same edge $e=[u, w]$. Let $L_{p}, L_{q}$ be two linear arc segments of $e$ such that $P \in$ $L_{p}, Q \in L_{q}$. The triangle inequality implies that $L_{p}$ and $L_{q}$ are not antipodal to each other, consequently the distance $d(P, Q)$ is either convex (if $L_{p}=L_{q}$ ), or linear (if $L_{p} \neq L_{q}$ ), and it is given by the length of subedge $[P, Q]$. By using the above notation $x=l(u, P)$ and $y=l(u, Q)$, we have $d(P, Q)=|x-y|$.

\section{Decomposing the 2-transfer covering problem}

Summarizing the previous section, for each pair of linear arc segments the distance $d(P, Q)$ between their respective points is either concave or convex, and its analytical expression can be computed. For this reason, the solution method is based on decomposing problem (2-TC) into a collection of independent subproblems (where each subproblem is the restriction of (2-TC) to a given pair of linear arc segments), and solving each of them via discretization of the solution set. To this end and for the sake of readability, we will describe the process in three phases: the first one is devoted to both decomposing the problem (2-TC) and grouping the subproblems into two cases: the concave and the convex case. The second phase deals with the subproblems of the first case, and finally in the last step we will study the subproblems of the second. These two cases will be described as follows.

To decompose the problem we first compute, in $O(|V||E|)$ time, the distance matrix between all pairs of nodes of the network. Then, for each edge $e \in E$ we obtain, and sort, the set $B_{e}$ (in $O(|V| \log |V|)$ time). At the end of this process we have, for each edge $e$ of the network, the ordered sequence $\mathcal{L}(e)$ of all linear arc segments of the edge. Besides, given a pair of different edges $e_{p}=\left[u_{p}, w_{p}\right]$ and $e_{q}=\left[u_{q}, w_{q}\right]$ we know whether or not two linear arc segments $L_{p} \in \mathcal{L}\left(e_{p}\right)$ and $L_{q} \in \mathcal{L}\left(e_{q}\right)$ are antipodal.

Let $\mathcal{L}=\bigcup_{e \in E} \mathcal{L}(e)$ be the set of all linear arc segments of the overall network. Thus problem (2-TC) is decomposed into a set of subproblems, where each of them is obtained by restricting the feasible space $\mathcal{N} \times \mathcal{N}$ to $L_{p} \times L_{q}$, with $L_{p}, L_{q} \in \mathcal{L}$. By imposing that $X_{1} \in L_{p}, X_{2} \in L_{q}$ we obtain the restricted problem:

$\max _{X_{1} \in L_{p}, X_{2} \in L_{q}} F\left(X_{1}, X_{2}\right):=\sum_{(i, j) \in C\left(X_{1}, X_{2}\right)} t_{i j}$

A solution to problem (2-TC) is found by solving the collection of all $O\left(|\mathcal{L}|^{2}\right)$ restricted problems (2-TCR) (in fact, $\frac{|\mathcal{L}|(|\mathcal{L}|-1)}{2}$ restricted problems by symmetry), and then selecting the best solution.

The classification of the restricted problem (2-TCR) is made according to the concavity of the distance $d\left(X_{1}, X_{2}\right)$, which is related to the antipodal character of $L_{p}, L_{q}$. More specifically, problem (2$\mathrm{TCR}$ ) is concave if the following condition holds:

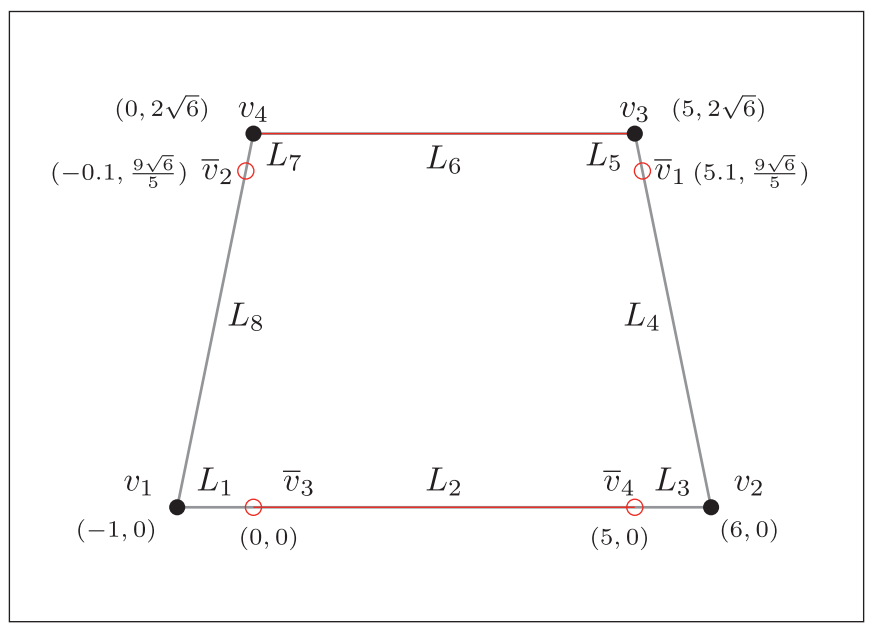

Fig. 4. Network with $V=\left\{v_{i}, i=1, \ldots, 4\right\}$, and linear arc segments $L_{p}, p=1, \ldots, 8$.

$L_{p} \in \mathcal{L}\left(e_{p}\right), L_{q} \in \mathcal{L}\left(e_{q}\right)$, with $e_{p} \neq e_{q}$, and $L_{p}, L_{q}$ are antipodal to each other.

For the remaining cases, the problem (2-TCR) is classified as convex.

\section{A procedure for discretizing the restricted problem (2-TCR)}

This section is devoted to studying the restricted problem (2-TCR) for a given pair $L_{p}, L_{q} \in \mathcal{L}$. Henceforth, we write $\left(X_{1}, X_{2}\right)$ $\in L_{p} \times L_{q}$ instead of $X_{1} \in L_{p}, X_{2} \in L_{q}$.

The strategy for solving (2-TCR) is based on identifying a Finite Dominating Set (FDS), that is, a finite set of points $\Omega \subset L_{p} \times$ $L_{q}$ containing an optimal solution. From this set, (2-TCR) becomes the problem $\max _{\left(X_{1}, X_{2}\right) \in \Omega} F\left(X_{1}, X_{2}\right)$. In order to describe the procedure for finding $\Omega$, the concave and convex cases are analyzed separately.

\subsection{The concave case}

This problem can be formulated from (2-TCR) by adding the assumption " $L_{p}, L_{q}$ are antipodal to each other", and it will be identified as (2-TCR) (1).

Each linear arc segment is a rectifiable subedge in which all distance functions $d\left(v_{i}, P\right)$ are linear. On the other hand, we have:

1. For each $A_{i} \in \mathcal{A}$, the Euclidean distance $\left\|A_{i}-X\right\|$ is convex when $X$ varies in any linear arc segment. In effect, this result follows straightforwardly from convexity theory (see Rockafeller, 1970), since if $f$ is a convex function in an open set $U$ then the restriction of $f$ to any interval (i.e., straight line segment) inside $U$ is convex.

2. When $\left(X_{1}, X_{2}\right) \in L_{p} \times L_{q}$, with $L_{p}, L_{q}$ antipodal to each other, the distance $\alpha d\left(X_{1}, X_{2}\right)$ is concave (Lemma 7), where $\alpha \in(0$, $1)$ is the speed factor.

Therefore both the function $h_{i j}^{+}\left(X_{1}, X_{2}\right)$ and $h_{i j}^{-}\left(X_{1}, X_{2}\right)$ are not convex on $L_{p} \times L_{q}$.

To illustrate some concepts and properties associated with this case, we will use the network with trapezoidal shape shown in Fig. 4. The lengths of the basis edges of the trapezoid are 7 and 5 , respectively, and the nodes $\left\{v_{i}, i=1, \ldots, 4\right\}$ are indicated in the figure with their corresponding coordinates. Likewise, each $\bar{v}_{i}$ represents the arc bottleneck point opposite to $v_{i}, i=1, \ldots, 4$. This figure also shows the partition of the network originated by the set of linear arc segments $\mathcal{L}=\left\{L_{p}, p=1, \ldots, 8\right\}$. In this case, $L_{2}=$ $\left[\bar{v}_{3}, \bar{v}_{4}\right]$ and $L_{6}=\left[v_{4}, v_{3}\right]$ are antipodal to each other. 

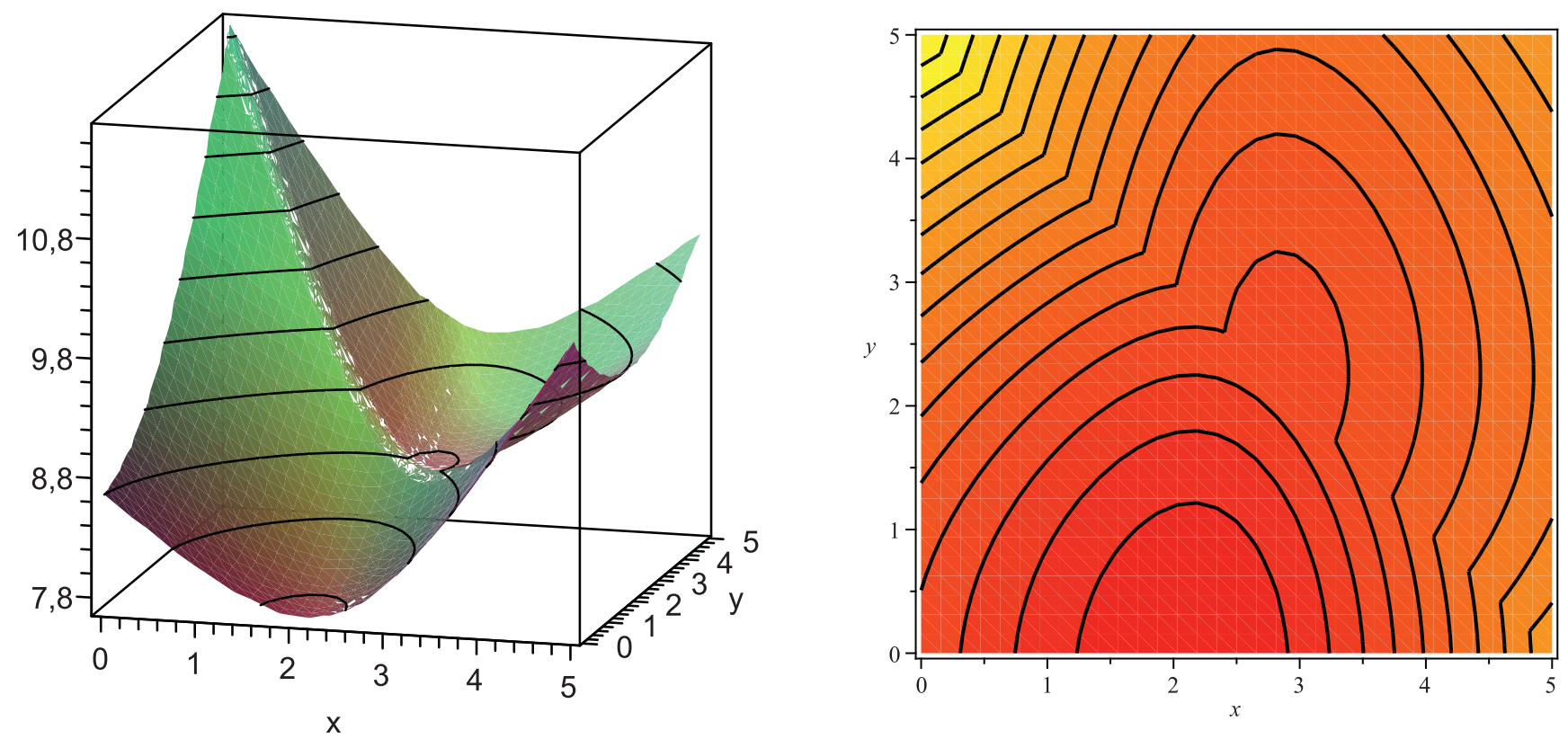

Fig. 5. Surface $h_{i j}^{+}\left(X_{1}, X_{2}\right)$ for $\alpha=0.3$ (left), and sets $H_{i j}^{+}(\eta)$ of such a surface (right).

In order to find an FDS for the problem, in the following we introduce the necessary definitions on the sublevel sets, from which the FDS is constructed.

\subsubsection{Characterizing the sublevel sets}

When $\left(X_{1}, X_{2}\right) \in L_{p} \times L_{q}$, and $\left\{L_{p}, L_{q}\right\}$ are antipodal to each other, the distance $d\left(X_{1}, X_{2}\right)$ is a concave function obtained from the lower envelope of two linear functions: $d_{a}\left(X_{1}, X_{2}\right)$ and $d_{b}\left(X_{1}\right.$, $\left.X_{2}\right)$. That is, $d\left(X_{1}, X_{2}\right)=\min \left\{d_{a}\left(X_{1}, X_{2}\right), d_{b}\left(X_{1}, X_{2}\right)\right\}$, where this expression is obtained from Lemma 7 by replacing $P$ and $Q$ by $X_{1}$ and $X_{2}$, respectively. More specifically, if $x=l\left(u_{p}, X_{1}\right)$ and $y=l\left(u_{q}, X_{2}\right)$, we have

$$
\begin{aligned}
d_{a}\left(X_{1}, X_{2}\right) & =x+d\left(u_{p}, u_{q}\right)+y, \text { and } d_{b}\left(X_{1}, X_{2}\right) \\
& =l\left(u_{p}, w_{p}\right)-x+d\left(w_{p}, w_{q}\right)+l\left(u_{q}, w_{q}\right)-y .
\end{aligned}
$$

If there is no confusion we will identify $X_{1}$ with $x$ and $X_{2}$ with $y$. Therefore, the travel distance $h_{i j}^{+}\left(X_{1}, X_{2}\right)$ of travel path $\left(A_{i}, X_{1}, X_{2}\right.$, $A_{j}$ ) can be expressed as

$h_{i j}^{+}\left(X_{1}, X_{2}\right)=\left\|A_{i}-X_{1}\right\|+\alpha \min \left\{d_{a}\left(X_{1}, X_{2}\right), d_{b}\left(X_{1}, X_{2}\right)\right\}+\left\|X_{2}-A_{j}\right\|$ and consequently,

$h_{i j}^{+}\left(X_{1}, X_{2}\right)=\min \left\{g_{i j}^{+, a}\left(X_{1}, X_{2}\right), g_{i j}^{+, b}\left(X_{1}, X_{2}\right)\right\}$,

where

$g_{i j}^{+, a}\left(X_{1}, X_{2}\right)=\left\|A_{i}-X_{1}\right\|+\alpha d_{a}\left(X_{1}, X_{2}\right)+\left\|X_{2}-A_{j}\right\|$

$g_{i j}^{+, b}\left(X_{1}, X_{2}\right)=\left\|A_{i}-X_{1}\right\|+\alpha d_{b}\left(X_{1}, X_{2}\right)+\left\|X_{2}-A_{j}\right\|$.

Since $d\left(X_{2}, X_{1}\right)=d\left(X_{1}, X_{2}\right)$, in a similar manner we can define the travel distance $h_{i j}^{-}\left(X_{1}, X_{2}\right)$ of travel path $\left(A_{i}, X_{2}, X_{1}, A_{j}\right)$ :

$h_{i j}^{-}\left(X_{1}, X_{2}\right)=\min \left\{g_{i j}^{-, a}\left(X_{1}, X_{2}\right), g_{i j}^{-, b}\left(X_{1}, X_{2}\right)\right\}$,

with $g_{i j}^{-, t}\left(X_{1}, X_{2}\right)=\left\|A_{i}-X_{2}\right\|+\alpha d_{t}\left(X_{1}, X_{2}\right)+\left\|X_{1}-A_{j}\right\|$, for $t \in\{a$, b).

For example, when $X_{1} \in L_{6}, X_{2} \in L_{2}$, Fig. 5 (left) shows the function $h_{i j}^{+}\left(X_{1}, X_{2}\right)$ on the network of Fig. 4 for $\alpha=0.3, A_{i}(2.5,6)$, $A_{j}(1,-4)$. Note the pagoda roof-shape of the surface.

Definition 8 (Sublevel Sets). Let $\left\{L_{p}, L_{q}\right\}$ be two linear arc segments antipodal to each other such that $\left(X_{1}, X_{2}\right) \in L_{p} \times L_{q}$. For $\eta \geq 0$, let us consider:
1. The $(\eta)$-sublevel set $H_{i j}^{+}(\eta)$ of function $h_{i j}^{+}\left(X_{1}, X_{2}\right)$, given by:

$H_{i j}^{+}(\eta)=\left\{\left(X_{1}, X_{2}\right) \in L_{p} \times L_{q}: h_{i j}^{+}\left(X_{1}, X_{2}\right) \leq \eta\right\}$.

Analogously, $H_{i j}^{-}(\eta)=\left\{\left(X_{1}, X_{2}\right) \in L_{p} \times L_{q}: h_{i j}^{-}\left(X_{1}, X_{2}\right) \leq \eta\right\}$.

2. For $t \in\{a, b\}$, the $(\eta)$-sublevel sets $G_{i j}^{+, t}(\eta)$ and $G_{i j}^{-, t}(\eta)$, of functions $g_{i j}^{+, t}\left(X_{1}, X_{2}\right)$ and $g_{i j}^{-, t}\left(X_{1}, X_{2}\right)$, respectively, given by:

$$
\begin{aligned}
& G_{i j}^{+, t}(\eta)=\left\{\left(X_{1}, X_{2}\right) \in L_{p} \times L_{q}: g_{i j}^{+, t}\left(X_{1}, X_{2}\right) \leq \eta\right\} \\
& G_{i j}^{-, t}(\eta)=\left\{\left(X_{1}, X_{2}\right) \in L_{p} \times L_{q}: g_{i j}^{-, t}\left(X_{1}, X_{2}\right) \leq \eta\right\} .
\end{aligned}
$$

For several $\eta$-values, Fig. 5 (right) displays the corresponding sublevel sets $H_{i j}^{+}(\eta)$ of the surface represented on the left-hand side of the figure.

For $t \in\{a, b\}$, the function $g_{i j}^{+, t}\left(X_{1}, X_{2}\right)$ is the sum of the convex term $\left\|A_{i}-X_{1}\right\|_{2}+\left\|A_{j}-X_{2}\right\|_{2}$ and the linear function $\alpha d_{t}\left(X_{1}, X_{2}\right)$. Similarly, $g_{i j}^{-, t}\left(X_{2}, X_{1}\right)$ is also the sum of a convex and a linear term. Therefore, the convexity of the sets $G_{i j}^{+, t}(\eta)$ and $G_{i j}^{-, t}(\eta)$, for $t \in$ $\{a, b\}$, is a straightforward consequence (see Boyd \& Vanderberghe, 2004).

From these definitions, and taking into account that if $h=$ $\min \{f, g\}$, the level set of $h$ is the union of the level sets of $f$ and $g$, we have:

Lemma 9. $H_{i j}^{+}(\eta)=G_{i j}^{+, a}(\eta) \cup G_{i j}^{+, b}(\eta)$, and $H_{i j}^{-}(\eta)=G_{i j}^{-, a}(\eta) \cup$ $G_{i j}^{-, b}(\eta)$

Consequently, both $H_{i j}^{+}(\eta)$ and $H_{i j}^{-}(\eta)$ are the union of two convex (but possibility not disjoint) sets. The following example shows that $G_{i j}^{+, a}(\eta) \cap G_{i j}^{+, b}(\eta) \neq \emptyset$, for some $\eta$-values, with $\eta<\left\|A_{i}-A_{j}\right\|$.

In Fig. 4, we have $\left\|A_{i}-A_{j}\right\|=\frac{\sqrt{409}}{2} \simeq 10.11$. Fig. 6(a) displays, for $\alpha=0.4$ and $\eta=10$, the boundaries of the sublevel sets $G_{i j}^{+, a}(\eta)$ and $G_{i j}^{+, b}(\eta)$. It can be observed that the intersection of both sets is not empty.

Proposition 10. For any $0 \leq \eta<\left\|A_{i}-A_{j}\right\|, H_{i j}^{+}(\eta) \cap H_{i j}^{-}(\eta)=\emptyset$, i.e., if there exists a path from $A_{i}$ to $A_{j}$ with length shorter than the Euclidean (planar) travel distance, then the order in which $X_{1}$ and $X_{2}$ are passed is uniquely determined. 
a

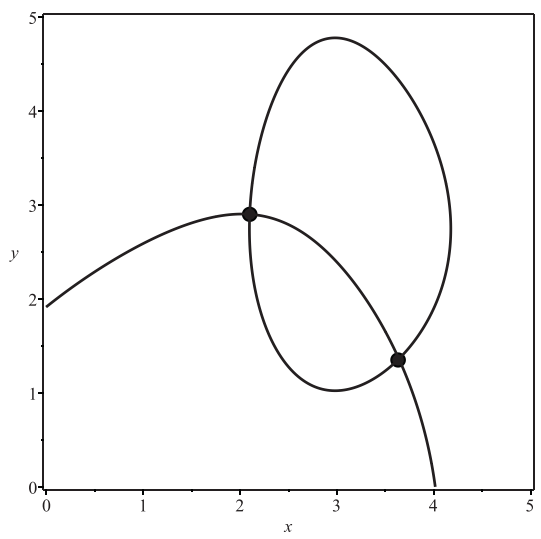

b

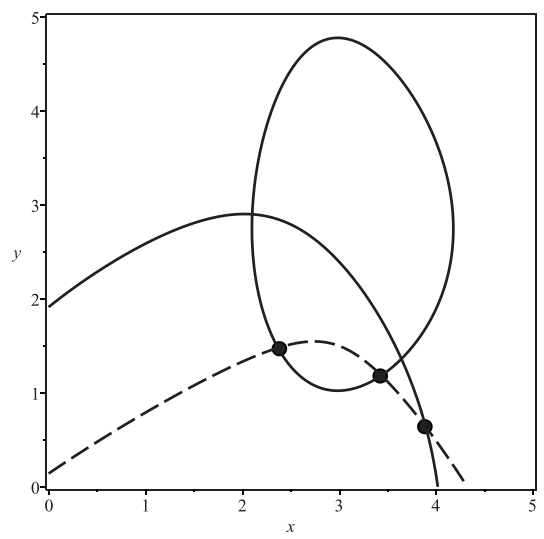

C

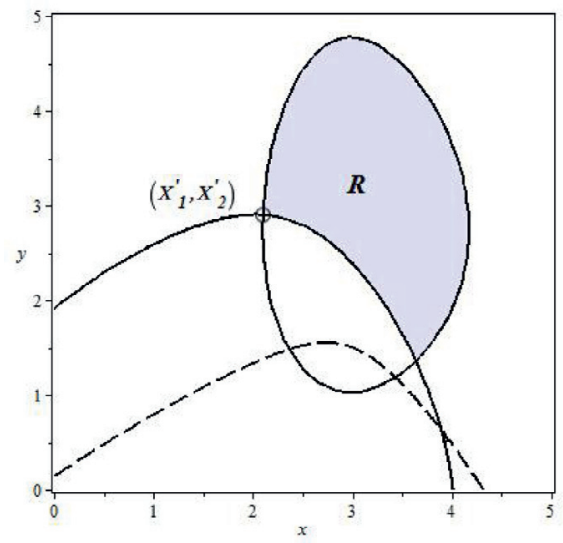

Fig. 6. (a): For $\widehat{d_{i j}}=10$, curves $\bar{G}_{i j}^{+, a}, \bar{G}_{i j}^{+, b}$, and set $\mathcal{Q}^{+}(i, j)=\bar{G}_{i j}^{+, a} \cap \bar{G}_{i j}^{+, b}$. (b): Set $\mathcal{P}((i, j),(k, r))$, for $\widehat{d_{k r}}=10.5$. (c): Vertex $\left(X_{1}^{\prime}, X_{2}^{\prime}\right)$ in the boundary of a region $R$.

Proof. Let $0<\eta<\left\|A_{i}-A_{j}\right\|$ and assume $H_{i j}^{+}(\eta) \cap H_{i j}^{-}(\eta) \neq \emptyset$. Then there exists $\left(X_{1}, X_{2}\right)$ such that

$\left\|A_{i}-X_{1}\right\|+\alpha d\left(X_{1}, X_{2}\right)+\left\|A_{j}-X_{2}\right\| \leq \eta$

$\left\|A_{i}-X_{2}\right\|+\alpha d\left(X_{1}, X_{2}\right)+\left\|A_{j}-X_{1}\right\| \leq \eta$.

Summing up and using that $d\left(X_{1}, X_{2}\right) \geq 0$ we obtain that

$\left\|A_{i}-X_{1}\right\|+\left\|A_{j}-X_{1}\right\|+\left\|A_{i}-X_{2}\right\|+\left\|A_{j}-X_{2}\right\| \leq 2 \eta$.

Due to the triangle inequality, we have that $\left\|A_{i}-A_{j}\right\| \leq \| A_{i}-$ $X_{k}\|+\| A_{j}-X_{k} \|$ for $k=1,2$. This gives us

$2\left\|A_{i}-A_{j}\right\| \leq\left\|A_{i}-X_{1}\right\|+\left\|A_{j}-X_{1}\right\|+\left\|A_{i}-X_{2}\right\|+\left\|A_{j}-X_{2}\right\| \leq 2 \eta$,

a contradiction to the assumption that $\eta<\left\|A_{i}-A_{j}\right\|$.

Let $\left\{L_{p}, L_{q}\right\}$ be antipodal to each other. For a given point $\left(X_{1}, X_{2}\right)$ $\in L_{p} \times L_{q}$, the objective value $F\left(X_{1}, X_{2}\right)=\sum_{(i, j) \in C\left(X_{1}, X_{2}\right)} t_{i j}$ quantifies the amount of weights of OD-pairs captured by such a point. Taking into account Definition 1 , let $H_{i j}$ be the set of points which cover the OD-pair $(i, j)$, given by

$H_{i j}=\left\{\left(X_{1}, X_{2}\right) \in L_{p} \times L_{q}: f_{i j}\left(X_{1}, X_{2}\right) \leq \widehat{d_{i j}}\right\}$.

The following result follows directly from the definition

Corollary 11. $\left(X_{1}, X_{2}\right) \in H_{i j}$ if and only if $(i, j) \in C\left(X_{1}, X_{2}\right)$.

In order to obtain $H_{i j}$ from the above defined sublevel sets, we replace the $\eta$-values by the specific acceptance level $\widehat{d_{i j}}$ associated with each OD-pair $(i, j)$.

Remark 12 (Notation). Given an acceptance level $0 \leq \widehat{d}_{i j}<\| A_{i}-$ $A_{j} \|_{2}$, for $t \in\{a, b\}$ let us denote $G_{i j}^{+, t}\left(\widehat{d_{i j}}\right)$ and $G_{i j}^{-, t}\left(\widehat{d_{i j}}\right)$ by $G_{i j}^{+, t}$ and $G_{i j}^{-, t}$, respectively. This abbreviated notation is also applied to the sets $H_{i j}^{+}\left(\widehat{d}_{i j}\right)$ and $H_{i j}^{-}\left(\widehat{d}_{i j}\right)$, which will be identified by $H_{i j}^{+}$and $H_{i j}^{-}$, respectively.

Corollary 13. For each OD-pair $(i, j), H_{i j}=H_{i j}^{+} \cup H_{i j}^{-}=G_{i j}^{+, a} \cup G_{i j}^{+, b} \cup$ $G_{i j}^{-, a} \cup G_{i j}^{-, b}$.

Proof. Since $f_{i j}\left(X_{1}, X_{2}\right)=\min \left\{h_{i j}^{+}\left(X_{1}, X_{2}\right), h_{i j}^{-}\left(X_{1}, X_{2}\right)\right\}=f_{j i}\left(X_{1}, X_{2}\right)$, the result follows from Lemma 9.

\subsubsection{Identifying a finite dominating set}

We now briefly comment on the role of $H_{i j}$ in the construction of a Finite Dominating Set (FDS). Recall that $C\left(X_{1}, X_{2}\right)=$ $\{(i, j), \ldots,(k, r)\}$ is the set of OD-pairs covered by $\left(X_{1}, X_{2}\right)$. Corollary 11 implies that $\left(X_{1}, X_{2}\right) \in H_{i j} \cap \ldots \cap H_{k r}$. For this reason, we next analyze the intersections of these sets, since such intersections will provide the points to be incorporated in the FDS, as we will see in the following. In fact, due to the absence of convexity of both $\mathrm{H}_{i j}^{+}$and $\mathrm{H}_{i j}^{-}$, we will focus the effort on selecting points from the boundaries of all these sets (as well as from their intersections), in order to guarantee that the selected points belong to all sets involved in the intersection.

Henceforth we will use the notation $\bar{G}$ (or $\bar{H}$ ), to identify the boundary or level curve of a set $G$ (or $H$ ). That is, for $t \in\{a, b\}$, we have

$\bar{G}_{i j}^{+, t}=\left\{\left(X_{1}, X_{2}\right) \in L_{p} \times L_{q}: g_{i j}^{+, t}\left(X_{1}, X_{2}\right)=\widehat{d_{i j}}\right\}$

$\bar{H}_{i j}^{+}=\left\{\left(X_{1}, X_{2}\right) \in L_{p}, L_{q}: h_{i j}^{+}\left(X_{1}, X_{2}\right)=\widehat{d_{i j}}\right\}$,

and analogously for $\bar{G}_{i j}^{-, t}$ and $\bar{H}_{i j}^{-}$. Clearly, for $\tau \in\{+,-\}, \bar{H}_{i j}^{\tau} \subseteq$ $\bar{G}_{i j}^{\tau, a} \cup \bar{G}_{i j}^{\tau, b}$, and $\bar{H}_{i j}^{\tau}=\bar{G}_{i j}^{\tau, a} \cup \bar{G}_{i j}^{\tau, b}$ if the set $G_{i j}^{\tau, a} \cap G_{i j}^{\tau, b}$ contains at most one point. Likewise

$\bar{H}_{i j}=\left\{\left(X_{1}, X_{2}\right) \in L_{p} \times L_{q}: f_{i j}\left(X_{1}, X_{2}\right)=\widehat{d_{i j}}\right\}$.

Since from Proposition $10, H_{i j}^{+} \cap H_{i j}^{-}=\emptyset$, we trivially conclude

Corollary 14. $\bar{H}_{i j}=\bar{H}_{i j}^{+} \cup \bar{H}_{i j}^{-}$.

As we have already seen, in order to construct the FDS of problem (2-TCR) (1), we will successively select several feasible points from these level curves and their intersections.

Lemma 15. Given two different OD-pairs $(i, j),(k, r)$, let $\mathcal{P}((i, j),(k, r)) \subset L_{p} \times L_{q}$ be the set of intersection points defined as follows:

$\mathcal{P}((i, j),(k, r))=\bigcup\left\{\left(\bar{G}_{i j}^{\tau, t} \cap \bar{G}_{k r}^{\tau^{\prime}, t^{\prime}}\right), \tau, \tau^{\prime} \in\{+,-\}, t, t^{\prime} \in\{a, b\}\right\}$.

Then, $\bar{H}_{i j} \cap \bar{H}_{k r} \subseteq \mathcal{P}((i, j),(k, r))$.

Proof. $\bar{H}_{i j}=\bar{H}_{i j}^{+} \cup \bar{H}_{i j}^{-} \subseteq\left(\bar{G}_{i j}^{+, a} \cup \bar{G}_{i j}^{+, b}\right) \cup\left(\bar{G}_{i j}^{-, a} \cup \bar{G}_{i j}^{-, b}\right)$, and a similar inclusion can be obtained for $\bar{H}_{k r}$. Therefore we can write

$$
\begin{gathered}
\bar{H}_{i j} \cap \bar{H}_{k r} \subseteq\left(\bar{G}_{i j}^{+, a} \cup \bar{G}_{i j}^{+, b} \cup \bar{G}_{i j}^{-, a} \cup \bar{G}_{i j}^{-, b}\right) \\
\bigcap\left(\bar{G}_{k r}^{+, a} \cup \bar{G}_{k r}^{+, b} \cup \bar{G}_{k r}^{-, a} \cup \bar{G}_{k r}^{-, b}\right) .
\end{gathered}
$$

Clearly, $\mathcal{P}((i, j),(k, r))$ is the right-hand side of this expression since $\mathcal{P}((i, j),(k, r))$ is obtained by intersecting each set of the first group with each set of the second group. This concludes the proof. 
Definition 16. For each OD-pair $(i, j)$, let $\mathcal{Q}(i, j)=\mathcal{Q}^{+}(i, j) \cup$ $\mathcal{Q}^{-}(i, j)$ be a set of feasible points of $\bar{H}_{i j}=\bar{H}_{i j}^{+} \cup \bar{H}_{i j}^{-}$, where for each $\tau \in\{+,-\}$ :

$$
\begin{aligned}
& \mathcal{Q}^{\tau}(i, j)= \begin{cases}\bar{G}_{i j}^{\tau, a} \cap \bar{G}_{i j}^{\tau, b}, & \begin{array}{r}
\text { if this intersection contains at least } \\
\text { one feasible point. } \\
\left\{X^{a}, X^{b}\right\},
\end{array} \\
\text { otherwise, where the points } X^{a} \text { and }\end{cases} \\
& X^{b} \text { are arbitrarily selected from } \\
& \bar{G}_{i j}^{\tau, a}, \bar{G}_{i j}^{\tau, b} \text {, respectively. }
\end{aligned}
$$

Clearly $\mathcal{Q}^{\tau}(i, j) \subset \bar{H}_{i j}^{\tau}$, for $\tau \in\{+,-\}$.

Fig. 6(a) and (b) shows some of these sets.

Fig. 6 is based on the network of Fig. 4, with $\alpha=0.4$. We have considered the points $A_{i}(2.5,6), A_{j}(1,-4), A_{k}(-2,-4.5)$ and $A_{r}(3$, 5.5). For $\widehat{d}_{i j}=10$ and $\widehat{d}_{k r}=10.5$, Fig. 6(a) shows the curves $\bar{G}_{i j}^{+, a}$ and $\bar{G}_{i j}^{+, b}$, as well as the corresponding set $\mathcal{Q}(i, j)=\bar{G}_{i j}^{+, a} \cap \bar{G}_{i j}^{+, b}$ containing two intersection points (in this case $H_{i j}^{-}=\emptyset$ ). Likewise, Fig. 6(b) displays the set of intersection points $\mathcal{P}((i, j),(k, r))$ containing three points. In this figure only a branch of the curve $\bar{G}_{k r}^{-, a}$ is inside the feasible domain.

Theorem 17. For the concave restricted problem (2-TCR)(1), let $\mathcal{Q}$ and $\mathcal{P}$ be the sets defined as follows

$\mathcal{Q}=\bigcup_{(i, j)} \mathcal{Q}(i, j), \quad \mathcal{P}=\bigcup_{(i, j) \neq(k, r)} \mathcal{P}((i, j),(k, r))$

Then, for any arbitrary point $X^{p q} \in L_{p} \times L_{q}$, the set $\Omega=\mathcal{Q} \cup \mathcal{P} \cup\left\{X^{p q}\right\}$ is an FDS for the problem (2-TCR)(1)

Proof. Let $\mathcal{G}=\bigcup_{(i, j)}\left\{\bar{G}_{i j}^{\tau, t}, \tau \in\{+,-\}, t \in\{a, b\}\right\} \subset L_{p} \times L_{q}$ be the collection of all level curves of the restricted problem (2-TCR)(1). From both Definition 8 and subsequent results, the collection $\mathcal{G}$ induces a partition $\Sigma$ of the feasible domain $L_{p} \times L_{q}$ into a set of regions $\left\{R_{s}, s \in I\right\}$ (where $I$ is an index set), such that both $F\left(X_{1}\right.$, $\left.X_{2}\right)$ and $C\left(X_{1}, X_{2}\right)$ are constant in the interior points of each region (see Fig. 6(c)). The possible changes in the objective function may only occur at the points on the boundary of each region. Therefore an FDS for problem (2-TCR)(1) can be constructed by selecting points from the set of boundaries $\left\{\bar{R}_{S}, s \in I\right\}$.

Given a region $R \in \Sigma$, we analyze the cases $R=L_{p} \times L_{q}$ and $R \subset L_{p} \times L_{q}$.

1. If $R=L_{p} \times L_{q}$, then $\mathcal{G}=\emptyset$. Hence, $F\left(X_{1}, X_{2}\right), C\left(X_{1}, X_{2}\right)$ are constant, for all $\left(X_{1}, X_{2}\right) \in L_{p} \times L_{q}$. Equivalently, any point $X^{p q}=\left(X_{1}, X_{2}\right) \in L_{p} \times L_{q}$ is optimal.

2. Otherwise, $R \subset L_{p} \times L_{q}$, and its boundary $\bar{R}$ contains a set of edges: pieces of level curves, and possibly some vertex which is a point shared by (at least) two different level curves (Fig. 6(c)). All points of an edge obtained from the level curve $\bar{G}_{i j}^{\tau, t} \in \mathcal{G}$ cover the OD-pair $(i, j)$. Therefore, given a set of edges incident to a vertex, such a vertex covers all OD-pairs associated with these edges. In other words: if $\left(X_{1}^{\prime}, X_{2}^{\prime}\right)$ is a vertex, then $F\left(X_{1}^{\prime}, X_{2}^{\prime}\right) \geq F\left(X_{1}, X_{2}\right)$, for any point $\left(X_{1}, X_{2}\right)$ of each edge incident to $\left(X_{1}^{\prime}, X_{2}^{\prime}\right)$. From this argument, all vertices of the partition $\Sigma$ are selected to be incorporated to the FDS. Additionally, if there is some $\bar{R}_{S}$ with none vertex, then $\bar{R}_{S}$ contains a single level curve, in which case an arbitrary point of such a curve is also added to the FDS.

In the following we prove that the FDS thus constructed is the set $\Omega$. In effect, a vertex $\left(X_{1}^{\prime}, X_{2}^{\prime}\right)$ of $\bar{R}$ is the intersection point of (at least) two level curves, and these curves can be obtained either from a single OD-pair $(i, j)$ or from (at least) two OD-pairs $(i, j),(k, r)$. (a) In the first case, Lemma 9 and Proposition 10 imply that $\left(X_{1}^{\prime}, X_{2}^{\prime}\right) \in \bar{G}_{i j}^{\tau, a} \cap \bar{G}_{i j}^{\tau, b}$. From Definition 16, this intersection is the set $\mathcal{Q}^{\tau}(i, j)$.

(b) Let assume two edges associated with the OD-pairs $(i$, $j)$ and $(k, r)$ are incident to $\left(X_{1}^{\prime}, X_{2}^{\prime}\right)$. This means that $\left(X_{1}^{\prime}, X_{2}^{\prime}\right)$ is obtained from the intersection $\bar{G}_{i j}^{\tau, t} \cap \bar{G}_{k r}^{\tau^{\prime}, t}$, for some $\tau, \tau^{\prime} \in\{+,-\}$ and $t, t^{\prime} \in\{a, b\}$, which implies that it belongs to the set $\mathcal{P}((i, j),(k, r))$.

Steps (a) y (b) are repeated with each vertex of the partition $\Sigma$. At the end of this process, all vertices of the case (b) are the set $\mathcal{P}$. On the other hand, an arbitrary point is selected from each $\bar{R}_{S}$ without vertices: these arbitrary points, together with all vertices of the case (a), are the set $\mathcal{Q}$. Finally, the arbitrary point $X^{p q}$ may be selected as one of points previously selected (in fact, $X^{p q}$ is necessary only if $\mathcal{G}=\emptyset$ ). This concludes the proof.

\subsection{The convex case}

This problem, identified by (2-TCR)(2), is formulated by adding the assumption that $\left\{L_{p}, L_{q}\right\}$ are not antipodal to each other to problem (2-TCR).

In this case, when $\left(X_{1}, X_{2}\right) \in L_{p} \times L_{q}$, from Lemma 7 and the subsequent reasoning, the distance $d\left(X_{1}, X_{2}\right)$ is convex. More specifically, we have

$d_{a}\left(\left(X_{1}, X_{2}\right)=d_{b}\left(X_{1}, X_{2}\right)=d\left(X_{1}, X_{2}\right)\right.$.

Consequently, all results of the previous section are valid for (2$\mathrm{TCR})(2)$ taking into account that we now have:

$H_{i j}^{+}=G_{i j}^{+, a}=G_{i j}^{+, b}$, and $H_{i j}^{-}=G_{i j}^{-, a}=G_{i j}^{-, b}$.

Therefore, from Proposition 10 and Corollary 13, both $H_{i j}^{+}$and $H_{i j}^{-}$ are convex and disjoint sets such that $H_{i j}=H_{i j}^{+} \cup H_{i j}^{-}$. Likewise, for this problem the set $\mathcal{Q}(i, j)$ of Definition 16 becomes the set $\mathcal{Q}(i, j)=\left\{X^{+}, X^{-}\right\}$, where $X^{+}=\left(X_{1}^{+}, X_{2}^{+}\right)$and $X^{-}=\left(X_{1}^{-}, X_{2}^{-}\right)$are points arbitrarily selected from $\bar{H}_{i j}^{+}$and $\bar{H}_{i j}^{-}$, respectively. And for each two different OD-pairs $(i, j),(k, r)$, the set $\mathcal{P}((i, j),(k, r))$ of Lemma 15 is now given by

$$
\begin{gathered}
\mathcal{P}((i, j),(k, r))=\bigcup_{(i, j) \neq(k, r)}\left(\bar{H}_{i j} \cap \bar{H}_{k r}\right)=\left\{\bar{H}_{i j}^{+} \cap \bar{H}_{k r}^{+}\right\} \cup\left\{\bar{H}_{i j}^{+} \cap \bar{H}_{k r}^{-}\right\} \\
\cup\left\{\bar{H}_{i j}^{-} \cap \bar{H}_{k r}^{+}\right\} \cup\left\{\bar{H}_{i j}^{-} \cap \bar{H}_{k r}^{-}\right\} .
\end{gathered}
$$

With these sets, Theorem 17 remains valid and establishes that $\Omega=\mathcal{Q} \cup \mathcal{P} \cup\left\{X^{p q}\right\}$ is a FDS for this problem.

Finally, since $C_{V}$ is a constant set, it follows straightforwardly:

Corollary 18. Let us consider that there exists a set of stations located on the nodes of the network. Then, $\Omega$ is also a FDS for the problem in which the set $C_{V}$ is excluded: $\max _{\left(X_{1}, X_{2}\right) \in L_{p} \times L_{q}} F\left(X_{1}, X_{2}\right):=$ $\sum_{(i, j) \in C\left(X_{1}, X_{2}\right) \backslash c_{V}} t_{i j}$.

\subsection{Example}

This example, based on the network of Fig. 4, illustrates the above procedure.

The existing demand is located at the points: $A_{1}(2.5,6)$, $A_{2}(3,5.5), A_{3}(5.5, \sqrt{6} / 10), A_{4}(2,-4.5)$, and $A_{5}(1,-4)$. The partition of the network originated by the set of linear arc segments $\mathcal{L}=\left\{L_{p}, p=1, \ldots, 8\right\}$ provides 36 different restricted problems on the pairs $\left\{\left\{L_{p}, L_{q}\right\}, p=1, \ldots, 7, q=p+1, \ldots, 8\right\} \cup\left\{\left\{L_{p}, L_{p}\right\}, p=\right.$ $1, \ldots, 8\}$, (the pairs $\left\{L_{p}, L_{q}\right\}$ and $\left\{L_{q}, L_{p}\right\}$ give rise to symmetric restricted problems with the same solution). Among all these pairs, $\left\{L_{2}, L_{6}\right\},\left\{L_{1}, L_{5}\right\}$ and $\left\{L_{3}, L_{7}\right\}$ are antipodal to each other, and the remaining pairs are not. 

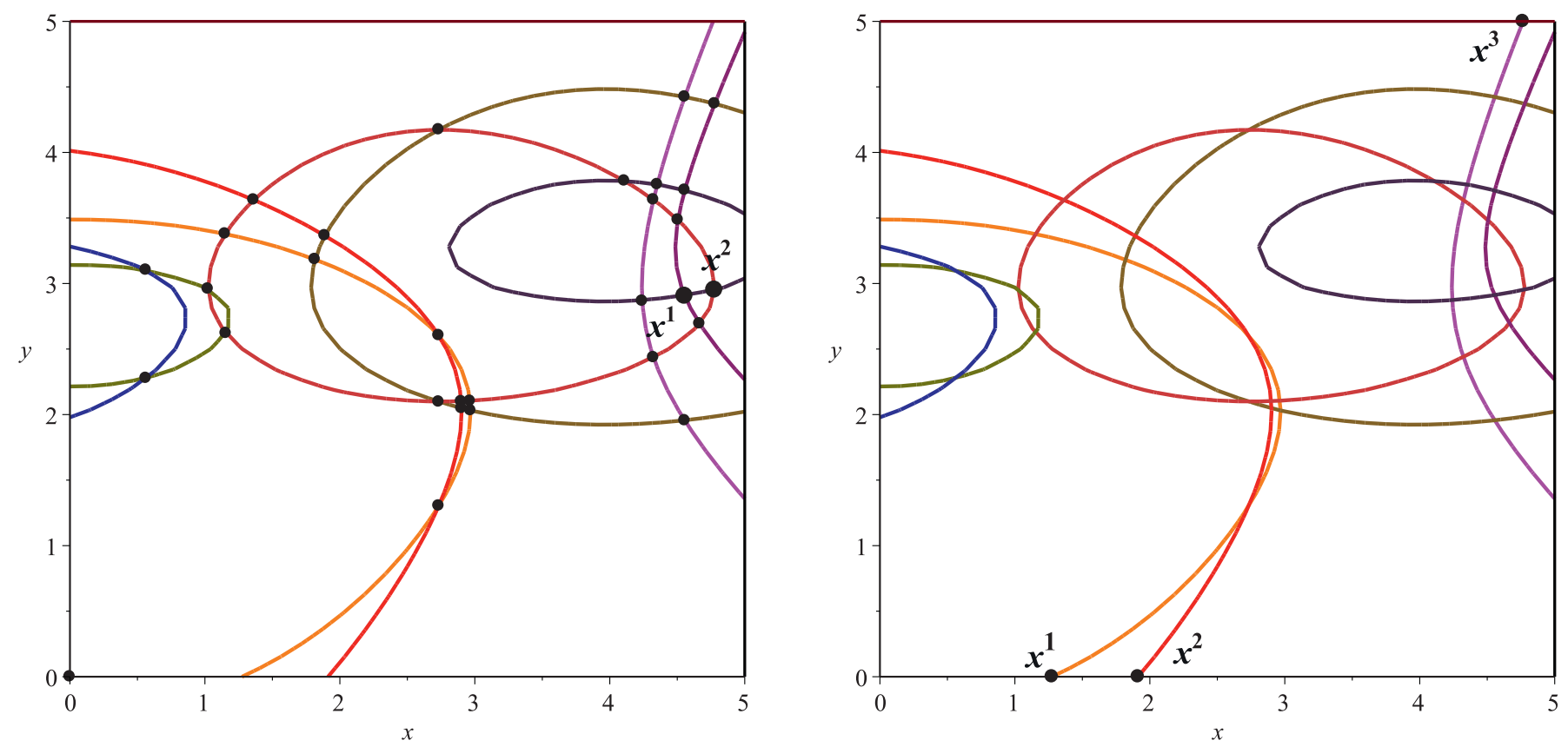

Fig. 7. Left: Collection $\mathcal{G}$ and partition $\Sigma$ for the concave restricted problem on $\left\{L_{2}, L_{6}\right\}$, and points of the FDS $\Omega$. Right: FDS for the subproblem (1) on $\left\{L_{2}\right.$, $\left.V\left(L_{6}\right)\right\}$.

We consider $\alpha=0.4$. The matrices $T=\left(t_{i j}\right)$ and $\widehat{D}=\left(\widehat{d}_{i j}\right)$ containing the weights and the acceptance levels for all OD-pairs, respectively, are given by

$T=\left(\begin{array}{ccccc}0 & 30 & 15 & 28 & 14 \\ 16 & 0 & 32 & 45 & 23 \\ 40 & 25 & 0 & 20 & 20 \\ 28 & 45 & 26 & 0 & 30 \\ 14 & 20 & 23 & 30 & 0\end{array}\right)$

$\widehat{D}=\left(\begin{array}{ccccc}0 & 0.5 & 6 & 10.2 & 10 \\ 0.5 & 0 & 5 & 9.2 & 8.5 \\ 6 & 5 & 0 & 5.5 & 5 \\ 10.2 & 9.2 & 5.5 & 0 & 1 \\ 10 & 8.5 & 5 & 1 & 0\end{array}\right)$

With this scenario we first have considered the concave restricted problem:

$\max _{\left(X_{1}, X_{2}\right) \in L_{2} \times L_{6}} F\left(X_{1}, X_{2}\right):=\sum_{(i, j) \in C\left(X_{1}, X_{2}\right)} t_{i j}$

Note that for $\left(X_{1}, X_{2}\right) \in L_{2} \times L_{6}, X_{1}=\left(x_{1}, 0\right) \in L_{2}$ and $X_{2}=$ $\left(x_{2}, 2 \sqrt{6}\right) \in L_{6}$, therefore each point $\left(X_{1}, X_{2}\right) \in \Omega$ is identified as $\boldsymbol{x}=\left(x_{1}, x_{2}\right)$. With this notation, $\boldsymbol{x}^{\boldsymbol{k}}=\left(x_{1}^{k}, x_{2}^{k}\right)$ denotes the $k$ th local solution $\left(X_{1}^{k}, X_{2}^{k}\right)$ of this subproblem (if any).

Fig. 7 (left) shows the FDS $\Omega$, constructed from all vertices of the partition $\Sigma$. The local solution for this subproblem is reached at both $\boldsymbol{x}^{\mathbf{1}}=(4.556013,2.90379)$ and $\boldsymbol{x}^{\mathbf{2}}=(4.776812,2.950328)$, with $\boldsymbol{x}^{\mathbf{1}} \in \mathcal{P}((2,3),(2,4))$ and $\boldsymbol{x}^{\mathbf{2}} \in \mathcal{P}((1,5),(2,4))$. For these points, the pairs covered and the objective value are $(1,3),(1,4)$, $(1,5),(2,3),(2,4)$ and 286 , respectively.

The following objective values (in decreasing order) are obtained from the convex restricted problems on $\left\{L_{3}, L_{6}\right\}$ and $\left\{L_{1}\right.$, $L_{6}$. Let $F_{p, q}^{*}$ be the optimum objective value of the restricted problem (2-TCR). Table 1 summarizes the results of the above three subproblems. The local solutions of the remaining restricted problems provide substantially lower objective values, and they have not been included in this table.

The solutions obtained from the restricted problem on $\left\{L_{2}, L_{6}\right\}$ are also global solutions for the 2-transfer problem on the over-
Table 1

Best optimum values for the 2-transfer problem.

\begin{tabular}{llll}
\hline $\begin{array}{l}\text { Restricted } \\
\text { problem }\end{array}$ & Local solutions & $C\left(X_{1}, X_{2}\right)$ & $F_{p, q}^{*}$ \\
\hline$\left\{L_{2}, L_{6}\right\}$ & $\begin{array}{l}\boldsymbol{x}^{\mathbf{1}}=(4.556013,2.90379) \\
\boldsymbol{x}^{\mathbf{2}}=(4.776812,2.950328)\end{array}$ & $(1,3),(1,4),(1,5),(2,3),(2,4)$ & 286 \\
$\left\{L_{3}, L_{6}\right\}$ & $\boldsymbol{x}^{\mathbf{3}}=(5.1,3.075407)$ & $(1,3),(1,4),(2,3),(2,4)$ & 258 \\
$\left\{L_{1}, L_{6}\right\}$ & $\boldsymbol{x}^{\mathbf{4}}=(0,2.2126927)$ & $(1,4),(1,5),(2,4),(2,5)$ & 217 \\
& $\boldsymbol{x}^{\mathbf{5}}=(0,3.1439062)$ & & \\
\hline
\end{tabular}

all network. The global optimum is $F^{*}=F_{2,6}^{*}=F\left(X_{1}^{*}, X_{2}^{*}\right)=286$, for $\left(X_{1}^{*}, X_{2}^{*}\right) \in\left\{\left(X_{1}^{k}, X_{2}^{k}\right), k=1,2\right\}$.

\section{The case of locating a single transfer point}

The above procedure also solves the case of locating one transfer point. In effect, as we have argued at the beginning of this paper, the formulation of the 1-transfer additional covering problem (1-TAC) is made under the hypothesis that all nodes of $V$ (or a subset of them) are already located transfer points. From this assumption, problem (1-TAC) can also be solved by adapting the procedure described for the 2-transfer restricted problem (2-TCR) to a set of subproblems dealing with the restriction of problem (1$\mathrm{TAC})$ to each linear arc segment $L_{p} \in \mathcal{L}$.

The 1-transfer problem (1-TAC) can be reformulated as follows:

$\max _{L_{p} \in \mathcal{L}} \max _{X \in L_{p}} F_{V}(X):=\sum_{(i, j) \in C(X) \backslash C_{V}} t_{i j}=\sum_{(i, j) \in\left(\bigcup_{X_{2} \in V} C\left(X, X_{2}\right)\right) \backslash C_{V}} t_{i j}$

where we have applied that $C(X)=\bigcup_{X_{2} \in V} C\left(X, X_{2}\right)$. The restriction of (1-TAC) to $L_{p} \in \mathcal{L}$ is:

$\max _{X \in L_{p}} F_{V}(X):=\sum_{(i, j) \in\left(\bigcup_{X_{2} \in V} C\left(X, X_{2}\right)\right) \backslash C_{V}} t_{i j}$

$(1-\mathrm{TACR})$ 
Let us consider the associated problem, in which $C_{V}$ is not excluded:

$\max _{X \in L_{p}} F_{V}(X):=\sum_{(i, j) \in \bigcup_{X_{2} \in V} C\left(X, X_{2}\right)} t_{i j}$

In the following we describe a procedure for finding an FDS for both problems.

For each $L \in \mathcal{L}$, let $V(L)=L \cap V$ denote the set of endpoints of $L$ which are nodes. Clearly, $0 \leq|V(L)| \leq 2$. We consider a set $\mathcal{L}_{p} \subseteq \mathcal{L}$ of linear arc segments such that the union of all node sets in the collection $\left\{V\left(L_{q}\right), L_{q} \in \mathcal{L}_{p}\right\}$ is the set $V$. There may be several sets $\mathcal{L}_{p}$ thus defined, we now indicate how to construct one of them.

\subsection{Constructing $\mathcal{L}_{p}$}

Let $\mathcal{L}^{\prime} \subset \mathcal{L}$ and $V^{\prime} \subseteq V$ be auxiliary sets. Initially, $\mathcal{L}^{\prime}=\mathcal{L} \backslash L_{p}$. Moreover, if $V\left(L_{p}\right)$ contains one node $w$ with degree 1 , then $\mathcal{L}_{p}=\left\{L_{p}\right\}$ and $V^{\prime}=V \backslash\{w\}$; otherwise $\mathcal{L}_{p}=\emptyset$ and $V^{\prime}=V$.

While $V^{\prime} \neq \emptyset$, repeat:

1. Select $L_{q} \in \mathcal{L}^{\prime}$ such that $V\left(L_{q}\right) \cap V^{\prime} \neq \emptyset$.

2. Update $\mathcal{L}_{p}:=\mathcal{L}_{p} \cup\left\{L_{q}\right\}$, and delete $L_{q}$ and $V\left(L_{q}\right)$ from $\mathcal{L}^{\prime}$ and $V^{\prime}$, respectively.

\subsection{Finding an FDS for the problem (1-TACR)}

To this end, we consider a collection of subproblems obtained from the restriction of (2-TCR) to each $V\left(L_{q}\right)$, with $L_{q} \in \mathcal{L}_{p}$. Thus, an FDS of (1-TACR) will be constructed from all FDS's of such a collection. This procedure is summarized in the following phases:

1. For each $L_{q} \in \mathcal{L}_{p}$, let $\Omega\left(L_{q}\right)$ denote an FDS of the subproblem

$$
\max _{X \in L_{p}, X_{2} \in V\left(L_{q}\right)} F\left(X, X_{2}\right):=\sum_{(i, j) \in C\left(X, X_{2}\right)} t_{i j}
$$

Obtaining $\Omega\left(L_{q}\right)$

Problem (2-TCR) with the additional restriction $X_{2} \in V\left(L_{q}\right)$ becomes (1). Therefore, the procedure for constructing an FDS of problem (2-TCR) can be applied to (1) as follows: Let us consider the collection $\mathcal{G}$ defined in Theorem 17 containing all level curves of (2-TCR), as well as the partition $\Sigma$ of $L_{p} \times L_{q}$ generated by $\mathcal{G}$, such that the changes in the objective function of (2-TCR) may only occur on the boundaries of $\Sigma$.

The feasible domain for subproblem (1) is $\left\{L_{p} \times X_{2}, X_{2} \in\right.$ $V\left(L_{q}\right)$ \}. By combining this domain with Theorem 17 we construct $\Omega\left(L_{q}\right)$ as follows:

$$
\Omega\left(L_{q}\right)=\bigcup_{v \in V\left(L_{q}\right)} \bigcup_{\bar{G}_{i j}^{\tau, t} \in \mathcal{G}}\left(\bar{G}_{i j}^{\tau, t} \cap\left\{X_{2}=v\right\}\right)
$$

In other words, $\Omega\left(L_{q}\right)$ is the set of intersection points of all level curves of $\mathcal{G}$ with the (at most two) segments of the feasible domain $\left\{L_{p} \times X_{2}, X_{2} \in V\left(L_{q}\right)\right\}$, (see Fig. 7, right). If this intersection is empty, for all $X_{2} \in V\left(L_{q}\right)$, then $\Omega\left(L_{q}\right)=$ $\left\{X^{p q}\right\}$, where $X^{p q}$ (the arbitrary point stated in Theorem 17), is any point selected from $\left\{L_{p} \times X_{2}, X_{2} \in V\left(L_{q}\right)\right\}$. From this theorem, $\Omega\left(L_{q}\right)$ is an FDS for the subproblem (1).

2. Let $\Omega_{p} \subset L_{p}$ be the points of the union set $\bigcup_{L_{q} \in \mathcal{L}_{p}} \Omega\left(L_{q}\right)$ in $L_{p}$, defined as:

$$
\Omega_{p}=\left\{X \in L_{p}:\left(X, X_{2}\right) \in \bigcup_{L_{q} \in \mathcal{L}_{p}} \Omega\left(L_{q}\right)\right\}
$$

Proposition 19. $\Omega_{p}$ is an FDS for the problem (1-TCR).

Proof. (a) Since $V=\bigcup_{L_{q} \in \mathcal{L}_{p}} V\left(L_{q}\right)$, we first prove that $\Omega_{p}$ does not depend on the collection $\mathcal{L}_{p}$ of linear arc segments, which is equivalent to prove that this independence is verified by $\bigcup_{L_{q} \in \mathcal{L}_{p}} \Omega\left(L_{q}\right)$

Let us consider a given node $v \in V$. From construction, any point $(X, v) \in \bigcup_{L_{q} \in \mathcal{L}_{p}} \Omega\left(L_{q}\right)$ belongs to a level curve of some boundary set $\bar{H}_{i j}$, which means that

$$
f_{i j}(X, v)=\left\|A_{i}-X\right\|+\alpha d(X, v)+\left\|v-A_{j}\right\|=\widehat{d}_{i j}
$$

Once $v$ is fixed, this function only depends on the point $X$ $\in L_{p}$. Equivalently the point $X \in L_{p}$ at which $f_{i j}(X, v)=\widehat{d}_{i j}$ is independent of the set $L_{q}$ selected, which implies $(X, v) \in$ $\Omega\left(L_{q}\right)$, for all $L_{q} \in \mathcal{L}_{p}$ such that $v \in V\left(L_{q}\right)$. Thus, $\bigcup_{L_{q} \in \mathcal{L}_{p}} \Omega\left(L_{q}\right)$ does not vary, for any selection of $\mathcal{L}_{p} \subseteq \mathcal{L}$ such that $V=$ $\bigcup_{L_{q} \in \mathcal{L}_{p}} V\left(L_{q}\right)$. Consequently, $\Omega_{p}$ is also independent of $\mathcal{L}_{p}$.

(b) The points of $\Omega_{p}$ generate a partition of $L_{p}$ into subintervals. We now prove that for each $X_{2} \in V$, the set $C\left(X, X_{2}\right)$ is constant in the interior points of each subinterval.

In effect, let $\Omega_{p}\left(L_{q}\right) \subset L_{p}$ be the points of $\Omega\left(L_{q}\right)$ in $L_{p}$, defined as

$\Omega_{p}\left(L_{q}\right)=\left\{X \in L_{p}:\left(X, X_{2}\right) \in \Omega\left(L_{q}\right)\right\}$

$\Omega_{p}\left(L_{q}\right)$ generates a partition of $L_{p}$ which we call a $q$ partition. On the other hand, the points of $\Omega\left(L_{q}\right)$ are the intersections of the collection $\mathcal{G}$ of level curves with the (at most) two segments $\left\{L_{p} \times X_{2}, X_{2} \in V\left(L_{q}\right)\right\}$. From Theorem 17, the properties of the partition $\Sigma$ generated by $\mathcal{G}$ imply that, when $\left(X_{1}, X_{2}\right) \in L_{p} \times L_{q}$, the possible changes of $C\left(X_{1}, X_{2}\right)$ may only occur on the boundaries of $\Sigma$. From construction, $\Omega\left(L_{q}\right)$ contains the points of the boundaries of $\Sigma$ placed on the above segments, therefore for each $X_{2} \in V\left(L_{q}\right)$ the set $C\left(X, X_{2}\right)$ is constant in the interior points of each subinterval of the $q$-partition, and the possible changes may only occur at the points of such a partition.

From definitions of both $\Omega_{p}$ and $\Omega_{p}\left(L_{q}\right)$ we have $\Omega_{p}=$ $\bigcup_{L_{q} \in \mathcal{L}_{p}} \Omega_{p}\left(L_{q}\right)$. This means that the partition defined by $\Omega_{p}$ incorporates all $q$-partitions. This fact and the above reasoning imply that for each $X_{2} \in V, C\left(X, X_{2}\right)$ is constant in the open subinterval limited by two consecutive points of $\Omega_{p}$.

Therefore the possible changes of $C(X)=\bigcup_{X_{2} \in V} C\left(X, X_{2}\right)$ may only occur on the points of $\Omega_{p}$, which implies that $\Omega_{p}$ is an FDS for the problem (1-TCR). This concludes the proof.

Corollary 20. $\Omega_{p}$ is also an FDS for the problem (1-TACR).

Remark 21. If $\Omega\left(L_{q}\right)=\left\{X^{p q}\right\}$ for all $L_{q} \in \mathcal{L}_{p}$, then $C\left(X, X_{2}\right)$ does not vary on $L_{p}$, for any $X_{2} \in V$, which means that $C(X)$ does not vary either. In such a case it is sufficient to select $X^{p} \in L_{p}$ arbitrarily and set $\Omega=\left\{X^{p}\right\}$.

\subsection{Computing the objective function of problem (1-TACR)}

Let us consider the points of $\Omega_{p}$ sorted in increasing order.

As it has been stated, $\Omega_{p}$ generates a partition of $L_{p}$ into intervals such that $C(X), F_{V}(X)$ and $\tilde{F}_{V}(X)$ are constant in the interior points of each interval. Additionally, $X^{\ell} \in \Omega_{p}$ if and only if $\left(X^{\ell}, X_{2}^{\ell}\right) \in \bar{H}_{i j}$, for some $X_{2}^{\ell} \in V$ and some OD-pair $(i, j)$. These facts allow the recursive computation of $F_{V}$ along the points of $\Omega_{p}$ as follows:

1. Initially, $\quad C\left(X^{\ell}\right)=\left\{(i, j):\left(X^{\ell}, X_{2}^{\ell}\right) \in \bar{H}_{i j}\right\}$, and $\quad F_{V}\left(X^{\ell}\right)=$ $\sum_{(i, j) \in C\left(X^{\ell}\right) \backslash C_{V}} t_{i j}$, for all $X^{\ell} \in \Omega_{p}$.

2. Let $X^{\ell}, X^{\ell+1}$ be two consecutive points of the sorted list $\Omega_{p}$. If $\left(X^{\ell}, X_{2}^{\ell}\right)$ and $\left(X^{\ell+1}, X_{2}^{\ell+1}\right)$ belong to $\bar{H}_{i j}$, then the objective value does not vary. Otherwise, $\left(X^{\ell}, X_{2}^{\ell}\right) \in \bar{H}_{i j}$ and 
Table 2

Set $\Omega\left(L_{6}\right)$ for problem (1) on $\left\{L_{2}, V\left(L_{6}\right)\right\}$.

\begin{tabular}{lll}
\hline Point $\boldsymbol{x}^{\ell}=\left(x_{1}^{\ell}, x_{2}^{\ell}\right)$ & $(i, j)$ & $C\left(X^{\ell}\right)$ \\
\hline $\boldsymbol{x}^{\mathbf{1}}=(1.276079,0)$ & $(1,4)$ & $(1,4),(1,5)$ \\
$\boldsymbol{x}^{2}=(1.913346,0)$ & $(1,5)$ & $(1,5)$ \\
$\boldsymbol{x}^{\mathbf{3}}=(4.7653359,5)$ & $(1,3)$ & $(1,3)$ \\
\hline
\end{tabular}

$\left(X^{\ell+1}, X_{2}^{\ell+1}\right) \in \bar{H}_{r s}$. In this case, the objective value is updated by evaluating whether $\left(X^{\ell}, X_{2}^{\ell}\right) \in H_{r s}$ or $\left(X^{\ell}, X_{2}^{\ell+1}\right) \in$ $H_{r s}$, (where $\left(X^{\ell}, X_{2}^{\ell}\right) \in H_{r s}$ if $f_{r s}\left(X^{\ell}, X_{2}^{\ell}\right) \leq \widehat{d}_{r s}$ ). If one of two cases occurs, then $C\left(X^{\ell}\right):=\left\{C\left(X^{\ell}\right) \cup\left\{C\left(X^{\ell+1}\right)\right\}\right.$, and $F_{V}$ is adequately updated.

In this process each point of $\Omega_{p}$ is compared with the following one, therefore it must be repeated twice along $\Omega_{p}$ : left to right and right to left, to compute the final objective value at each point.

\subsection{Example}

The construction of $\Omega\left(L_{q}\right)$ is shown in this example, which uses the same network, demand points and notation as in the previous example. For the 1-transfer problem restricted to $L_{2}$, the set $\mathcal{L}_{2}=$ $\left\{L_{1}, L_{3}, L_{6}\right\}$ gives the collection $\left\{V\left(L_{1}\right), V\left(L_{3}\right), V\left(L_{6}\right)\right\}$, whose union is $V$. Furthermore, $C_{V}=\emptyset$.

Fig. 7 (right) shows the FDS for the subproblem (1) when $X_{1} \in$ $L_{2}$ and $X_{2} \in V\left(L_{6}\right)=\left\{v_{3}, v_{4}\right\}$. The feasible domain for this subproblem is given by the boundaries $L_{2} \times v_{4}$ and $L_{2} \times v_{3}$ (i.e., the segments $x_{2}=0$ and $x_{2}=5$, respectively). To construct $\Omega\left(L_{6}\right)$ we only need to compute the intersections of the level curves with such boundaries.

For the subproblem (1) on $\left\{L_{2}, V\left(L_{6}\right)\right\}$, Table 2 shows the points of $\Omega\left(L_{6}\right)$, the OD-pairs $(i, j)$ whose associated level curves originate these intersections, and the corresponding sets $C\left(X^{\ell}\right)$.

Moreover, $\Omega\left(L_{q}\right)=\emptyset$ for the subproblems on both $\left\{L_{2}, V\left(L_{1}\right)\right\}$ and $\left\{L_{2}, V\left(L_{3}\right)\right\}$. Thus the set $\Omega_{2}=$ $\{(1.276079,0),(1.913346,0),(4.7653359,0)\} \subset L_{2} \quad$ is an FDS for the problem (1-TACR) on $L_{2}$.

Finally, problem (1-TACR) on $L_{6}$ provides the solution to the 1transfer problem on the overall network, which is reached at the points $(2.461131,2 \sqrt{6}),(2.97537,2 \sqrt{6}) \in L_{6}$, with objective value equal 217 , and the same set $\{1,4),(1,5),(2,4),(2,5)\}$ of covered OD-pairs.

\section{The 2-transfer additional covering problem}

The restriction of problem (2-TAC) to a given pair of linear arc segments $L_{p}, L_{q} \in \mathcal{L}$ is formulated as follows:

$$
\max _{\left(X_{1}, X_{2}\right) \in L_{p} \times L_{q}} F_{A}\left(X_{1}, X_{2}\right):=\sum_{(i, j) \in\left(C\left(X_{1}, X_{2}\right) \cup C\left(X_{1}\right) \cup C\left(X_{2}\right)\right) \backslash C_{V}} t_{i j}
$$

The strategy for discretizing the solution set is based on obtaining the FDS of the restricted problems (2-TCR) and (1-TACR), respectively. These sets together which the level curves from which they have been obtained will be combined in order to derive an FDS for (2-TACR). We now describe the elements of this process.

1. For a given pair $L_{p}, L_{q} \in \mathcal{L}$, let $\mathcal{G}$ and $\Sigma$ be the collection of level curves and the partition of $L_{p} \times L_{q}$, respectively, stated in Theorem 17 for the restricted problem (2-TCR). From this Theorem, both $F\left(X_{1}, X_{2}\right)$ and $C\left(X_{1}, X_{2}\right)$ are constant in the interior points of each region of such a partition.
2. Let $\Omega_{p}$ and $\Omega_{q}$ be the FDS of the problem (1-TACR) on $L_{p}$ and $L_{q}$, respectively. The vertical and horizontal segment lines through the points of $\Omega_{p}$ and $\Omega_{q}$ give rise to a grid $\Gamma$ which partitions $L_{p} \times L_{q}$ on cells, such that for $k=1,2$, both $F_{V}\left(X_{k}\right)$ and $C\left(X_{k}\right)$ are constant on the interior points of each cell.

3. Finally, let $\Sigma \cup \Gamma$ be the partition of $L_{p} \times L_{q}$ obtained by overlapping $\Sigma$ and $\Gamma$. From construction, possible changes in the objective function of (2-TACR) only take place at the points of the boundary of each region of $\Sigma \cup \Gamma$.

Let $\Omega_{p} \times \Omega_{q}$ be the set of the corner points of the grid $\Gamma$, and let $\Omega_{\gamma}=\mathcal{G} \cap \Gamma$ be the set containing the intersection points of the level curves in $\mathcal{G}$ with the segment lines of $\Gamma$. The above arguments and the fact that $C_{V}$ is a constant set yield the union of the sets $\Omega, \Omega_{p} \times \Omega_{q}$, and $\Omega_{\gamma}$, is an FDS for problem (2-TACR).

Corollary 22. The set $\Omega_{A}=\Omega \cup\left(\Omega_{p} \times \Omega_{q}\right) \cup \Omega_{\gamma}$ contains some optimal solution for both the restricted problem (2-TACR) and the associated restricted problem in which $C_{V}$ is not excluded.

\subsection{Some insights on evaluating the objective function}

Evaluating the objective function at each point $\left(X_{1}, X_{2}\right) \in \Omega_{A}$ requires computing $C\left(X_{1}, X_{2}\right)$ and $C\left(X_{k}\right), k=1,2$.

1. The algorithm for solving (2-TCR) provides $C\left(X_{1}, X_{2}\right)$, for all $\left(X_{1}, X_{2}\right) \in \Omega$. However, for the points in $\Omega_{p} \times \Omega_{q} \cup \Omega_{\gamma}$, the sets $C\left(X_{1}, X_{2}\right)$ are constructed by checking each OD-pair.

2. On the other hand, for all $\left(X_{1}, X_{2}\right) \in \Omega_{p} \times \Omega_{q}$, the sets $C\left(X_{k}\right)$, $k=1,2$ can be known by means of the algorithm for solving (1-TACR). Such an algorithm will be described subsequently. The knowledge of $C\left(X_{k}\right)$ for the points in $\Omega_{p} \times \Omega_{q}$ allows the computation of $C\left(X_{k}\right),(k=1,2)$, for all $\left(X_{1}, X_{2}\right) \in \Omega \cup \Omega_{\gamma}$. In effect, each point $\left(X_{1}, X_{2}\right) \in \Omega_{\gamma}$ belongs to some line segment of $\Gamma$, from which we can obtain $C\left(X_{k}\right), k=1,2$, as follows: suppose that $\left(X_{1}, X_{2}\right) \in\left[X_{1}^{\ell}, X_{1}^{\ell+1}\right] \times X_{2}^{\kappa}$, where $X_{1}^{\ell}$, $X_{1}^{\ell+1}$ are two consecutive points of $\Omega_{p}$, and $X_{2}^{\kappa} \in \Omega_{q}$. Thus, $C\left(X_{2}\right)=C\left(X_{2}^{\kappa}\right)$, and $C\left(X_{1}\right) \in\left\{C_{V}\left(X_{1}^{\ell}\right), C\left(X_{1}^{\ell+1}\right)\right\}$. The computational effort is spent for inserting $X_{1}$ in the sorted list $\Omega_{p}$. This argument can also be extended to any point $\left(X_{1}, X_{2}\right) \in$ $\Omega$ : since $\left(X_{1}, X_{2}\right)$ belongs to some cell of the grid $\Gamma$, it is only necessary to insert $X_{1}$ and $X_{2}$ in the sorted lists $\Omega_{p}$ and $\Omega_{q}$, respectively.

\section{Algorithms and complexity}

Assuming that some technical questions (such as the bidimensional representation of the restricted problem by means of a parametrization of linear arc segments) have been previously obtained, the algorithms for solving the three restricted problems analyzed are based on progressively constructing their FDS, and then evaluating the corresponding objective function on the points of the FDS for finally selecting the best solution.

We start with a preprocessing phase in which the ordered sequence $\mathcal{L}(e)$ of all linear arc segments of each edge $e$ is computed. At the end of such a phase we can know if two linear arc segments $L_{p} \in \mathcal{L}\left(e_{p}\right)$ and $L_{q} \in \mathcal{L}\left(e_{q}\right)$ are whether or not antipodal to each other. In the case of having an antipodal pair $\left\{L_{p}, L_{q}\right\}$, the distance $d\left(X_{1}, X_{2}\right)$, for $\left(X_{1}, X_{2}\right) \in L_{p} \times L_{q}$ can be established from Lemma 7.

\subsection{The 2-transfer covering problem (2-TC)}

The solution procedure for both the concave and the convex case selects the global solution as the best of local solutions obtained from the collection of restricted problems. 
ALGORITHM FOR THE CONCAVE RESTRICTED PROBLEM (2-TCR)(1)

1. Set $\Omega:=\emptyset$.

2. For each OD-pair $(i, j)$, do

(a) Obtain the level curves $\bar{G}_{i j}^{+, a}, \bar{G}_{i j}^{+, b}, \bar{G}_{i j}^{-, a}$, and $\bar{G}_{i j}^{-, b}$. For each $\tau \in\{+,-\}$, do

i. If $\bar{G}_{i j}^{\tau, a} \cup \bar{G}_{i j}^{\tau, b} \neq \emptyset$, then compute $\mathcal{Q}^{\tau}(i, j)=$ $\bar{G}_{i j}^{\tau, a} \cap \bar{G}_{i j}^{\tau, b}$.

(1) If $\mathcal{Q}^{\tau}(i, j) \neq \emptyset$, then $\Omega:=\Omega \cup \mathcal{Q}^{\tau}(i, j)$.

(2) Otherwise, set $\Omega:=\Omega \cup\left\{X^{a}, X^{b}\right\}$, where $X^{a}, X^{b}$, are arbitrarily selected points from $\bar{G}_{i j}^{\tau, a}, \bar{G}_{i j}^{\tau, b}$, respectively.

(3) For each $\left(X_{1}, X_{2}\right) \in \mathcal{Q}^{\tau}(i, j)$, initialize $C\left(X_{1}, X_{2}\right)=\{(i, j),(j, i)\}$.

3. For each different OD-pairs $(i, j)$ and $(k, r)$, do

(a) Obtain $\mathcal{P}((i, j),(k, r))$, the set of intersection points of all pairs of level curves $\left\{\bar{G}_{i j}^{\tau, t}, \bar{G}_{k r}^{\tau^{\prime}, t^{\prime}}\right\}$, for $\tau, \tau^{\prime} \in\{+,-\}$ and $t, t^{\prime} \in\{a, b\}$.

(b) If $\mathcal{P}((i, j),(k, r)) \neq \emptyset$, then

i. For each $\left(X_{1}, X_{2}\right) \in \mathcal{P}((i, j),(k, r))$,

compute $C\left(X_{1}, X_{2}\right)=\{(i, j),(j, i),(k, r),(r, k)\}$

ii. Set $\Omega:=\Omega \cup \mathcal{P}((i, j),(k, r))$.

4. If $\Omega=\emptyset$, select arbitrarily $X^{p q} \in L_{p} \times L_{q}$. Initialize $C\left(X^{p q}\right)=\emptyset$, and set $\Omega=\left\{X^{p q}\right\}$.

5. For each $\left(X_{1}, X_{2}\right) \in \Omega$, do

(a) For each OD-pair $(i, j)$, do

i. If $\left(X_{1}, X_{2}\right) \in H_{i j}$, then

update $C\left(X_{1}, X_{2}\right):=C\left(X_{1}, X_{2}\right) \cup\{(i, j),(j, i)\}$, and $F\left(X_{1}, X_{2}\right)$.

6. Select as a solution a point $\left(X_{1}^{*}, X_{2}^{*}\right)$ for which $F\left(X_{1}^{*}, X_{2}^{*}\right)=$ $\max _{\left(X_{1}, X_{2}\right) \in \Omega} F\left(X_{1}, X_{2}\right)$ (there may be several solutions).

To discuss the resulting complexity we first analyze the cardinality of the sets $\mathcal{Q}, \mathcal{P}$, composing the FDS $\Omega$. We have previously pointed out that, as it is usual in origin-destination problems, henceforth we will use $N=O\left(n^{2}\right)$ to denote the number of OD-pairs in order to reflect the complexity as a function of $N$ (instead of as a function of the number $n$ of isolated demand points).

In this process, the main computational effort of the first four steps is spent in computing the $O\left(N^{2}\right)$ sets $\mathcal{P}((i, j),(k, r))$. Each $\bar{G}_{i j}^{\tau, t}$ is the boundary curve of the convex set $G_{i j}^{\tau, t}$, which is the sublevel set of the convex function $g_{i j}^{\tau, t}(\cdot)$. The algebraic structure of these functions allows us to establish (by means of Bezout's theorem), that for $\tau, \tau^{\prime} \in\{+,-\}$ and $t, t^{\prime} \in\{a, b\}$, the number of intersection points of two different curves $\bar{G}_{i j}^{\tau, t}, \bar{G}_{k r}^{\tau^{\prime}, t^{\prime}}$ is (upper) bounded by 12 (see Kirwan, 1992; Körner et al., 2014). Since each $\mathcal{P}((i, j),(k, r))$ is obtained from the intersection of (at most) $4^{2}=16$ pairs of curves, the complexity of $\mathcal{P}$ (the total number of intersection points) is $O\left(N^{2}\right)$. From the same argument, the cardinality of $\mathcal{Q}$ (computed in step 2) is also $O\left(N^{2}\right)$, consequently $|\Omega|$ $\in O\left(N^{2}\right)$. This implies that Step 5, in which for each point $\left(X_{1}, X_{2}\right)$ $\in \Omega$ the associated set $C\left(X_{1}, X_{2}\right)$ and the objective value is computed, requires $O\left(N^{3}\right)$ time. This gives a final complexity of $O\left(N^{3}\right)$ for solving the concave restricted problem (2-TCR)(1).

This algorithm also solves the convex restricted problem (2TCR)(2) by introducing some slight modifications in steps 2 and 3:

2. For each OD-pair $(i, j)$, do

(a) Obtain the level curves $\bar{H}_{i j}^{+}$and $\bar{H}_{i j}^{-}$. For each $\tau \in\{+,-\}$, do

i. If $\bar{H}_{i j}^{\tau} \neq \emptyset$, then

(1) Set $\Omega:=\Omega \cup\left\{X^{\tau}\right\}$, where $X^{\tau}=\left(X_{1}^{\tau}, X_{2}^{\tau}\right)$ is an arbitrary point in $\bar{H}_{i j}^{\tau}$.
(2) Initialize $C\left(X^{\tau}\right)=\{(i, j),(j, i)\}$.

3. For each different OD-pairs $(i, j)$ and $(k, r)$, do

(a) Compute $\mathcal{P}((i, j),(k, r))$, the set of all intersection points obtained from $\bar{H}_{i j}^{\tau} \cap \bar{H}_{k r}^{\tau^{\prime}}$, for $\tau, \tau^{\prime} \in\{+,-\}$.

(b) If $\mathcal{P}((i, j),(k, r)) \neq \emptyset$, then

i. For each $\left(X_{1}, X_{2}\right) \in \mathcal{P}((i, j),(k, r))$,

compute $C\left(X_{1}, X_{2}\right)=\{(i, j),(j, i),(k, r),(r, k)\}$.

ii. Set $\Omega:=\Omega \cup \mathcal{P}((i, j),(k, r))$.

The above modifications do not reduce the complexity of $O\left(N^{3}\right)$ obtained for the concave problem, since for the convex case we also have $O\left(N^{2}\right)$ sets $P((i, j),(k, r))$.

These reasonings imply that the restricted problem (2-TCR) can be solved in $O\left(N^{3}\right)$ time (independently of the case: concave or convex). Taking into account that there are at most $|V||E|$ linear arc segments in the network, the number of pairs of linear arc segments is $O\left(|V|^{2}|E|^{2}\right)$, which is the number of restricted problems (2-TCR) to be solved. This finally gives a complexity of $O\left(|V|^{2}|E|^{2} N^{3}\right)$ time for solving the 2-transfer problem (2-TC) on the overall network.

\subsection{The 1-transfer additional covering problem (1-TAC)}

With some modifications, the above algorithms can be applied to the restricted problem (1-TACR). We first assume that $C_{V}$ has been computed in a previous phase, which takes $O\left(N|V|^{2}\right)$ time.

Each level curve $\bar{G}_{i j}^{\tau, t}$ contributes with at most four points (if it is a closed curve) placed on the boundaries $L_{p} \times\left\{X_{2}: X_{2} \in\right.$ $V\left(L_{q}\right)$ \}, which implies that $\Omega\left(L_{q}\right)$, the FDS of (1), has a complexity of $O(N)$. From construction, $\left|\mathcal{L}_{p}\right|=O(|V|)$, therefore $\Omega_{p}$, the FDS of (1-TACR), has $O(|V| N)$ points.

Once these points have been sorted (in $O(|V| N \max \{\log |V|$, $\log N\}$ ) time), the objective value can be recursively computed from a point to the following one in $O\left(\left|C_{V}\right|\right)$ time. In summary, the final complexity for solving (1-TACR) is $O\left(|V| N \max \left\{\left|C_{V}\right|, \log |V|, \log N\right\}\right)$.

ALGORITHM FOR THE RESTRICTED PROBLEM (1-TACR)

1. Set $\Omega_{p}:=\emptyset$, and construct $\mathcal{L}_{p}$.

2. For each $L_{q} \in \mathcal{L}_{p}$ do

(a) Set $\Omega\left(L_{q}\right):=\emptyset$.

(b) For each $\mathrm{O} / \mathrm{D}$ pair $(i, j)$ do

i. Obtain the level curves $\bar{G}_{i j}^{+, a}, \bar{G}_{i j}^{+, b}, \bar{G}_{i j}^{-, a}$, and $\bar{G}_{i j}^{-, b}$.

ii. For $\tau \in\{+,-\}, t \in\{a, b\}$ :

Compute $\bar{G}_{i j}^{\tau, t} \cap\left\{X_{2}=v\right\}$ for all $v \in V\left(L_{q}\right)$, and update $\Omega\left(L_{q}\right)$.

(c) Update $\Omega_{p}:=\Omega_{p} \cup\left\{X:\left(X, X_{2}\right) \in \Omega\left(L_{q}\right)\right\}$.

3. If $\Omega_{p}=\emptyset$, then choose any point $X^{p} \in L_{p}$, and set $\Omega_{p}:=\left\{X^{p}\right\}$.

4. Sort $\Omega_{p}$, and compute the objective function along $\Omega_{p}$.

5. Select $X^{*} \in \Omega_{p}$ such that $F_{V}\left(X^{*}\right)=\max _{X \in \Omega_{p}} F_{V}(X)$.

From the above discussion, the 1-transfer additional covering problem (1-TAC) can be solved on the overall network in $O\left(|V|^{2}|E| N \max \left\{\left|C_{V}\right|, \log |V|, \log N\right\}\right)$ time.

\subsection{The 2-transfer additional covering problem (2-TAC)}

For finding $\Omega_{A}$, the FDS stated in Corollary 22, we start by computing $\Omega, \Omega_{p}$ and $\Omega_{q}$, for which we use the algorithms for problems (2-TCR) and (1-TACR), respectively. Regarding the complexities already established, we have $|\Omega| \in O\left(N^{2}\right)$ and $\left|\Omega_{p} \times \Omega_{q}\right| \epsilon$ $O\left(|V|^{2} N^{2}\right)$. On the other hand, each level curve of the collection $\mathcal{G}$ is convex on $L_{p} \times L_{q}$ and intersects with (at most) $O(|V| N)$ segment lines of the grid $\Gamma$, which implies that $\Omega_{\gamma}$ has $O\left(|V| N^{2}\right)$ points. This leads to a complexity of $O\left(|V|^{2} N^{2}\right)$ for the set $\Omega_{A}$. 


\section{ALGORITHM FOR THE RESTRICTED PROBLEM (2-TACR)}

1. Obtain $\mathcal{G}, \Omega$, and $C\left(X_{1}, X_{2}\right), \forall\left(X_{1}, X_{2}\right) \in \Omega$, by algorithm for problem (2-TCR).

Set $\Omega_{A}:=\Omega$.

2. Obtain $\Omega_{p}, \Omega_{q}$, and the sets $C\left(X_{k}\right),(k=1,2)$, for $\left(X_{1}, X_{2}\right) \in$ $\Omega_{p} \times \Omega_{q}$, by algorithm for problem (1-TACR).

Update $\Omega_{A}:=\Omega_{A} \cup\left(\Omega_{p} \times \Omega_{q}\right)$.

3. Set $\Omega_{\gamma}:=\emptyset$. For each level curve $\bar{G}_{i j}^{\tau, t} \in \mathcal{G}$ do

Compute $\bar{G}_{i j}^{\tau, t} \cap\left\{X_{1}=X_{1}^{\ell}\right\}$ and $\bar{G}_{i j}^{\tau, t} \cap\left\{X_{2}=X_{2}^{\kappa}\right\}$, for all $X_{1}^{\ell} \in$ $\Omega_{p}, X_{2}^{\kappa} \in \Omega_{q}$.

Update $\Omega_{\gamma}$, and $\Omega_{A}$.

4. For each $\left(X_{1}, X_{2}\right) \in \Omega \cup \Omega_{\gamma}$, do

Insert $X_{1}$ and $X_{2}$ into $\Omega_{p}$ and $\Omega_{q}$, respectively, and compute $C\left(X_{k}\right), k=1,2$.

5. For each $\left(X_{1}, X_{2}\right) \in \Omega_{p} \times \Omega_{q} \cup \Omega_{\gamma}$, do

For each OD-pair $(i, j)$, check whether $\left(X_{1}, X_{2}\right) \in H_{i j}$.

Update $C\left(X_{1}, X_{2}\right)$ adequately.

6. For each $\left(X_{1}, X_{2}\right) \in \Omega_{A}$, compute $F_{A}\left(X_{1}, X_{2}\right)$.

7. Select the best solution as a solution for the problem (there may be several solutions).

Steps 1 and 2 of this algorithm takes $O\left(N^{3}\right)$ and $O(|V| N$ $\max \{\log |V|, \log N\}$ time, respectively. Inserting a point into the sorted lists $\Omega_{p}$ and $\Omega_{q}$ requires $O(|V| N)$ time and, from Section 7.1, this is the complexity of Step 4. Taken into account that $\Omega_{p} \times \Omega_{q}$ is the set with the largest cardinality and $\left|C_{V}\right| \leq N$, the complexity of Steps 5 and 6 is $O\left(|V|^{2} N^{3}\right)$ time, which also is the final complexity for solving (2-TACR).

There are $O\left(|V|^{2}|E|^{2}\right)$ restricted problems, therefore the 2transfer additional covering problem (2-TAC) can be solved on the overall network in $O\left(|V|^{4}|E|^{2} N^{3}\right)$ time.

Remark 23. Although from a theoretical point of view the computational analysis of these problems deals with the worst-case complexity, in practise only a few OD-pairs are involved in each subproblem. In effect, given $L_{p}, L_{q}$, the pair $(i, j)$ can be excluded if $\min \left\{\left\|A_{i}-L_{p}\right\|+\left\|A_{j}-L_{q}\right\|,\left\|A_{j}-L_{p}\right\|+\left\|A_{i}-L_{q}\right\|\right\} \geq \widehat{d_{i j}}$, where $\|A-S\|$ is the Euclidean distance between the point $A$ and the segment $S$. Likewise, a set of linear arc segments could be removed, which leads to an improvement of the average complexity.

\section{Conclusions and further research}

Given a set of origin-destination pairs in the Euclidean plane, the problem of locating one or two transfer points on a high-speed network embedded in the plane under different OD-pairs covering objectives has been analyzed and solved in this paper.

The first problem studied seeks to locate two transfer points so that the amount of weights of the covered pairs is maximized. Given the lack of convexity of the mixed planar-network distances our approach is based on a decomposition of the network into smaller regions such that the travel distances between them are either concave or convex. This decomposition gives rise to two different restricted problems which have been analyzed and, for each of them, a finite dominating set of polynomial size has been derived. Hence, the problem can be solved by a polynomial time algorithm. Moreover, this solution method also represents a general approach for solving the remaining problems treated in this paper, as it has been described in the mechanism for adapting the general strategy to each case. Indeed, this method could also be extended to problems with more transfer points, although it is predictable that the complexity increases dramatically.

On the other hand, the methodology proposed here does not prejudge the assessment of whether one or two transfer points must be located, since this question involves a decision-making process in which additional considerations would be taken into account. From a strategical point of view, locating 2-transfer points guarantees better coverage in a long-term approach, however, in short-term decisions, it may be suitable to locate first a single transfer point and, at a later stage, to determine the convenience of locating the second one.

A way of making the model more realistic is to introduce acceleration and deceleration between nodes as well as dwell times associated with these points. This problem deserves further research. Actual or Manhattan distances are more appropriate in some cases. Whereas the counterpart problems deserve to be studied, they clearly are beyond the scope of this paper. We note that since the computational complexity of the corresponding problem of locating more than two transfer points is high, other methodologies such as the Big Cube Small Cube algorithm (Schöbel \& Scholz, 2010) could be useful for this extension. Another line of research could be oriented to considering a set $\mathcal{F}$ of forbidden regions (contained in the high-speed system) in which it is not possible to locate facilities. The forbidden regions arise when, for example, zones of the network pass under a river, or cross a natural park, etc. The algorithm presented in this paper can be adapted for dealing with this model in the following manner: both deleting from $\Omega$ those points that lie inside some forbidden region and adding finite intersections between $\mathcal{F}$ and the level curves of all sublevel sets.

Finally, a generalization of the model can result by incorporating other objectives (such as minimizing the sum of all distances between covered pairs, or maximizing the sum of differences between the acceptance level and the mixed travel distances, or by introducing other criterion associated to some costs). This multiobjective formulation possibly will yield optimal points placed in the interior of some sublevel sets, which provide a wide understanding of the role of solutions in order to select a more realistic optimal decision.

\section{Acknowledgment}

This work was partially supported by Ministerio de Economía y Competitividad (Spain)/FEDER under projects MTM2012-37048 and MTM2015-67706-P, and by Junta de Andalucía (Spain)/FEDER under excellence project P10-FQM-5849.

\section{References}

Boyd, S. P., \& Vanderberghe, L. (2004). Convex optimization. Cambridge University Press.

Carrizosa, E., Harbering, J., \& Schöbel, A. (2016). Minimizing the passengers' traveling time in the stop location problem. Journal of the Operational Research Society. doi:10.1057/jors.2016.3.

Church, R. L., \& ReVelle, C. S. (1974). The maximal covering location problem. Papers in Regional Science, 32, 101-118.

Contreras, I., \& Fernández, E. (2012). General network design: A unified view of combined location and network design problems. European Journal of Operational Research, 219, 680-697.

Hamacher, H., Liebers, A., Schöbel, A., Wagner, D., \& Wagner, F. (2001). Locating new stops in a railway network. Electronic Notes Theoretical Computers Science, 50, $1-11$.

Hooker, J. J., Garfinkel, R. S., \& Chen. , C. K. (1991). Finite dominating sets for network location problems. Operations Research, 39, 100-118.

Kirwan, F. (1992). Complex algebraic curves. Cambridge University Press.

Körner, M.-C., Mesa, J. A., Perea, F., Schöbel, A., \& Scholz, D. (2014). A maximum trip covering location problem with alternative mode of transportation on tree networks and segments. TOP, 22, 227-253.

Laporte, G., \& Mesa, J. A. (2015). The design of rapid transit networks. In G. Laporte, S. Nickel, \& F. F. Saldanha da Gama (Eds.), Location science: Chapter 22 (pp. 581-594).

Laporte, G., Mesa, J. A., \& Ortega, F. A. (2000). Optimization methods for the planning of rapid transit systems. European Journal of Operational Research, 122, $1-10$

Laporte, G., Mesa, J. A., \& Ortega, F. A. (2002). Locating stations on rapid transit lines. Computers \& Operations Research, 29, 741-759. 
Laporte, G., Mesa, J. A., Ortega, F. A., \& Sevillano, I. (2005). Maximizing trip coverage in the location of a single rapid transit line alignment. Annals of Operations Research, 136, 49-61.

Laporte, G., Mesa, J. A., \& Perea, F. (2014). Adding a new station and a road link to a road-rail network in the presence of modal competition. Transportation Research $B, 68,1-16$.

Murawski, I., \& Church, R. L. (2009). Improving accessibility to rural health services: The maximal covering network improvement problem. Socio Economic Planning Sciences, 43, 102-110.

Repolho, H. M., Antunes, A. P., \& Church, R. L. (2013). Optimal location of railway stations: The lisbon-porto high-speed rail line. Transportation Science, 47, 330-343.

Repolho, H. M., Church, R. L., \& Antunes, A. P. (2010). Optimum location of motorway interchanges: User's perspective. Journal of Transportation Engineering, 136, 956-963.
Rockafeller, R. T. (1970). Convex analysis. Princeton University Press.

Schöbel, A. (2005). Locating stops along bus or railway lines. a bicriteria problem. Annals of Operations Research, 136, 211-227.

Schöbel, A., Hamacher, H., Liebers, A., \& Wagner, D. (2009). The continuous stop location problem in public transportation networks. Asia-Pacific Journal of Operational Research, 26, 13-30.

Schöbel, A., \& Scholz, D. (2010). The big cube small cube solution method for multidimensional facility location problems. Computers \& Operations Research, 37, 115-122.

Vuchic, V. R. (1969). Rapid transit interstation spacings for maximum number of passengers. Transportation Science, 3, 214-232.

Vuchic, V. R., \& Newell, G. F. (1968). Rapid transit interstation spacing for minimum travel time. Transportation Science, 2, 303-309 